\title{
Non-Conforming Curved Finite Element Schemes for Time-Dependent Elastic-Acoustic Coupled Problems
}

\author{
Ángel Rodríguez-Rozas ${ }^{\mathrm{a}, \mathrm{b}, \mathrm{c}}$, Julien Diaz ${ }^{\mathrm{a}}$ \\ ${ }^{a}$ Inria Bordeaux-Sud-Ouest, Équipe-Projet Magique-3D IPRA-LMA, Université de Pau et des Pays de \\ l'Adour, BP 1155, 64013 Pau Cedex, France \\ ${ }^{b} B C A M$ - Basque Center for Applied Mathematics, Mazarredo 14, E48009, Bilbao, Basque Country - \\ Spain. \\ ${ }^{c}$ University of the Basque Country (UPV/EHU), Department of Applied Mathematics, Statistics, and \\ Operational Research, Leioa, Spain
}

\begin{abstract}
High-order numerical methods for solving time-dependent acoustic-elastic coupled problems are introduced. These methods, based on Finite Element techniques, allow for a flexible coupling between the fluid and the solid domain by using non-conforming meshes and curved elements.

Since characteristic waves travel at different speeds through different media, specific levels of granularity for the mesh discretization are required on each domain, making impractical a possible conforming coupling in between. Advantageously, physical domains may be independently discretized in our framework due to the non-conforming feature. Consequently, an important increase in computational efficiency may be achieved compared to other implementations based on conforming techniques, namely by reducing the total number of degrees of freedom. Differently from other non-conforming approaches proposed so far, our technique is relatively simpler and requires only a geometrical adjustment at the coupling interface at a preprocessing stage, so that no extra computations are necessary during the time evolution of the simulation.

On the other hand, as an advantage of using curvilinear elements, the geometry of the coupling interface between the two media of interest is faithfully represented up to the order of the scheme used. In other words, higher order schemes are in consonance with higher order approximations of the geometry. Concerning the time discretization, we analyze both explicit and implicit schemes. These schemes are energy conserving and, for the explicit case, the stability is guaranteed by a CFL condition.

In order to illustrate the accuracy and convergence of these methods, a set of representative numerical tests are presented.
\end{abstract}

Email addresses: angel.rodriguez@ehu.es (Ángel Rodríguez-Rozas), julien.diaz@inria.fr (Julien Diaz) 


\section{Introduction}

The development of efficient numerical methods for the simulation of waves at the interface between fluid and solid is an issue that is raised by many applications. Let us in particular mention the simulation of earthquakes in oceanic crust or offshore seis-

5 mic imaging. There are also medical applications, such as the simulation of ultrasound propagation in biological tissues, or the detection of solid objects immersed in a fluid.

Many methods have been proposed to solve this issue in harmonic domain, let us mention the BEM/FEM type method, whose principle consists in using a Boundary Element Method to discretize the fluid and a Finite Element Method for the solid [1,

10 2, 3, 4, 5]; plane waves based methods, using the Partition of the Unity Method [6], Ultraweak Formulation [7] or Discontinuous Enrichment Methods [8]; or Discontinuous Galerkin Methods [9].

Concerning transient waves simulation, one can use Finite Differences Methods, based for instance on the Virieux scheme [10], setting the velocity of S-wave to zero in the fluid.

15 However, this often leads to instabilities or to spurious waves in the fluid. Moreover, Finite Differences are not very convenient to deal with topography and Robertsson proposed a strategy to deal with irregular interface [11]. Finite Element Methods are much more adapted to deal with topography and, among them, Spectral Element Methods (SEM) are very popular in the Geophysical community, since they allow naturally for the use

zo of explicit time schemes [12, 13, 14, 15, 16]. In [17], Komatitsch, Barnes and Tromp applied the SEM successfully to fluid-structure problems. Another solution methodology, based on Discontinuous Galerkin Methods (DGM) has been proposed in [18], allowing for non-conforming meshes.

The use of non-conforming meshes is necessary to obtain efficient numerical schemes

25 since the velocities of the waves in the fluid and in the solid may strongly differ. Hence, the wavelengths and the space steps required to fulfill the number of points per wavelength in each region are often very different. This non-conformity can be handled using Mortar Element Method [19, 20, 21, 22, 23], but this require the introduction of an additional unknown, representing the trace of the solution on the interface. The SEM can be easily

so adapted to deal with non-conforming meshes, as it has been shown for instance in [24] and in $[25,26]$, where the authors also proposed a local time stepping strategy in order to adapt the time steps to the two different space steps.

A second issue is the modeling of curved interfaces and many of the above methods can be easily extended to handle curved elements, see for instance [7, 9]. However, the

35 design of efficient numerical methods involving both non-conforming meshes and curved interfaces is still an open issue. In [27, 28, 29], Jaiman et al proposed a Combined Interface Boundary Condition scheme by imposing higher-order interface corrections. The drawback of this scheme is the additional computation of the correction at each coupling time step. A very efficient alternative, based on Discontinuous Galerkin Method

40 has been proposed in [30]. It is however restricted to the case where one the interface (usually the fluid one) is a submesh of the other one. In [31], proposed a to handle curved non conforming interfaces for sommation-by-part (SBP) finite difference methods.

In this work we propose a more general solution methodology, where the two domains can be meshed independently, up to some minor restrictions that we present below. We

${ }_{45}$ apply this methodology to transient problems discretized by classical Finite Element Method and using a classical Leap-Frog scheme. However, since our strategy is based on 
the meshing of the domain, it can be easily extended to harmonic problems, other types of Finite Element Methods, such as SEM or DGM, and other time schemes. It can also be applied to other multiphysics problems involving the coupling of subdomains with

so particular physical features which impose very different levels of granularity regarding their corresponding computational mesh

The outline of this paper is as follows. The model problem is presented in Sec. 2 and its corresponding weak formulation in Sec. 3. The non-conforming technique is presented in Sec. 4 and the details concerning its implementation are presented in Sec. 5.

${ }_{55}$ The discretization in time is presented in Sec. 6, where the positivity and conservation of the energy is carefully analyzed for both explicit and implicit schemes. Finally, in Sec. 7 we present numerical results validating the accuracy and convergence of our method with a reference test. In the same section, we also report its efficiency both in terms of execution time and memory usage.

\section{6о 2. Coupled system of equations and boundary conditions}

The domain $\Omega$ modeling the acoustic-elastic isotropic coupled problem of interest is divided into two subdomains, one corresponding to the fluid (acoustic) part and the other to the solid part, denoted by $\Omega_{f}$ and $\Omega_{s}$, respectively (see Fig. 1). The coupling boundary between these two sub-domains is denoted by $\Gamma_{i}$.

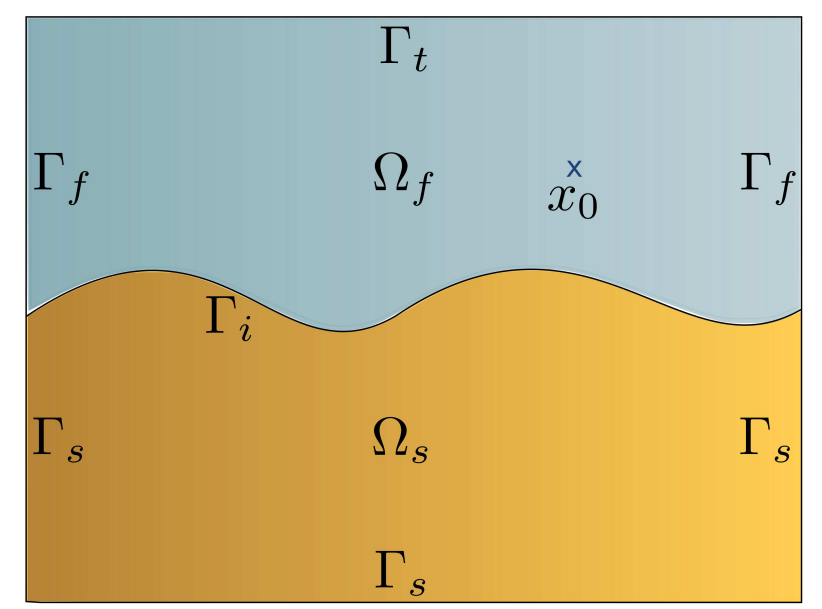

Figure 1: Domain problem with a curved coupling interface, $\Gamma_{i}$. The point source located at $\mathbf{x}_{0}$ is labeled as ' $\times$ '.

65 The wave propagation problem with open boundaries under consideration has to be solved computationally in a given truncated domain with a delimited boundary, $\Gamma=\Gamma_{f} \cup \Gamma_{s}$, where an artificial boundary condition must be imposed. Although several sophisticated solutions do exist in the literature which may be appropriate when considering high-order schemes, a first-order absorbing boundary condition (ABC) is chosen

7o for this work, for the sake of simplicity. On the top boundary of the acoustic subdomain, $\Gamma_{t}$, a free-condition is imposed. 
As for the system of equations, several formulations may alternatively be considered. Among them, a symmetric formulation that simplifies considerably energy estimates introduced later on is considered in this work. Such formulation consists of a fluid

75 pressure $(q)$ formulation for the fluid part and a velocity formulation for the elastic structure (v). Finally, the full mathematical continuous problem of interest reads as follows

$$
\begin{aligned}
\frac{1}{\rho_{f} c_{F}^{2}} \frac{\partial^{2} q}{\partial t^{2}}-\nabla \cdot\left(\frac{1}{\rho_{f}} \nabla q\right) & =f(t) \delta_{\mathbf{x}_{0}}, & & \text { in } \Omega_{f}, \\
\rho_{s} \frac{\partial^{2} \mathbf{v}}{\partial t^{2}}-\nabla \cdot \underline{\underline{\sigma}}(\mathbf{v}) & =0, & & \text { in } \Omega_{s}, \\
\underline{\underline{\sigma}}(\mathbf{v}) \cdot \mathbf{n}_{\mathbf{s}}-\frac{\partial q}{\partial t} \mathbf{n}_{\mathbf{f}} & =0, & & \text { on } \Gamma_{i}, \\
\rho_{f} \frac{\partial \mathbf{v}}{\partial t} \cdot \mathbf{n}_{\mathbf{s}}-\nabla q \cdot \mathbf{n}_{\mathbf{f}} & =0, & & \text { on } \Gamma_{i}, \\
q & =0, & & \text { on } \Gamma_{t}, \\
\frac{1}{c_{F}} \frac{\partial q}{\partial t}+\nabla q \cdot \mathbf{n}_{\mathbf{f}} & =0, & & \text { on } \Gamma_{f}, \\
\rho_{s} B \frac{\partial \mathbf{v}}{\partial t}+\underline{\underline{\sigma}}(\mathbf{v}) \cdot \mathbf{n}_{\mathbf{s}} & =0, & & \text { on } \Gamma_{s},
\end{aligned}
$$

where the external force $f(t) \delta_{\mathbf{x}_{0}}$ represents a point source in space located at $\mathbf{x}_{\mathbf{0}}, f(t)$ is the source time function, $c_{F}$ is the wave speed on the fluid part, $\mathbf{n}_{\mathbf{f}}$ and $\mathbf{n}_{\mathbf{s}}$ are the exterior unit normal to their respective boundaries, $\partial \Omega_{f}$ and $\partial \Omega_{s}$. The tensors

$$
\sigma_{i j}(\mathbf{v})=\lambda \nabla \cdot \mathbf{v} \delta_{i j}+2 \mu \epsilon_{i j}(\mathbf{v})
$$

and

$$
\epsilon_{i j}(\mathbf{v})=\frac{1}{2}\left(\frac{\partial v_{i}}{\partial x_{j}}+\frac{\partial v_{j}}{\partial x_{i}}\right)
$$

are, respectively, the classical Cauchy and stress tensors typically defined in a homogeneous and isotropic elastic media. The symmetric, positive-definite matrix $B$ appearing

so in $(1 \mathrm{~g})$ is defined as

$$
B:=\left(\begin{array}{cc}
c_{P} n_{s_{1}}^{2}+c_{S} n_{s_{2}}^{2} & \left(c_{P}-c_{S}\right) n_{s_{1}} n_{s_{2}} \\
\left(c_{P}-c_{S}\right) n_{s_{1}} n_{s_{2}} & c_{P} n_{s_{1}}^{2}+c_{S} n_{s_{2}}^{2}
\end{array}\right),
$$

where $c_{P}$ and $c_{S}$ are the compression and the shear wave velocity, respectively, with $c_{P}=\sqrt{\frac{\lambda+2 \mu}{\rho_{s}}}$ and $c_{S}=\sqrt{\frac{\mu}{\rho_{s}}}$.

\section{Weak formulation}

It is well known that the Finite Element Method (FEM) is one of the most general and ${ }_{85}$ flexible numerical methods for solving partial differential equations, specially whenever 
the definition of the problem of interest includes a curved domain and complex couplings. The classical FEM is chosen in this work due to its proven generality, robustness and simplicity regarding details of implementation. However, the non-conforming method that we introduce in this paper could easily be extended to other variants of FEM, such

so as Spectral Element Method or Discontinuous Galerkin Method and/or multiphysics problems.

Before describing the spatial and temporal discretization, we proceed now introducing the weak formulation of the coupled problem defined in (1) which reads as follows: $\forall t \in$ $(0, T]$, find $\mathbf{v}=\mathbf{v}(t) \in V$ and $q=q(t) \in Q$ such that

$$
\begin{aligned}
& \int_{\Omega_{s}} \rho_{s} \frac{\partial^{2} \mathbf{v}}{\partial t^{2}} \cdot \mathbf{w}_{\mathbf{s}} d \Omega+\int_{\Omega_{s}} \lambda \nabla \cdot \mathbf{v} \nabla \cdot \mathbf{w}_{\mathbf{s}} d \Omega+\int_{\Omega_{s}} 2 \mu \sum_{i, j=1}^{2} \epsilon_{i, j}(\mathbf{v}) \epsilon_{i, j}\left(\mathbf{w}_{\mathbf{s}}\right) d \Omega_{s} \\
& +\int_{\Gamma_{s}} \rho_{s}\left(B \frac{\partial \mathbf{v}}{\partial t}\right) \cdot \mathbf{w}_{\mathbf{s}} d \Gamma-\int_{\Gamma_{i}} \frac{\partial q}{\partial t} \mathbf{n}_{\mathbf{f}} \cdot \mathbf{w}_{\mathbf{s}} d \Gamma=0, \\
& \int_{\Omega_{f}} \rho_{f}^{-1} c_{F}^{-2} \frac{\partial^{2} q}{\partial t^{2}} w_{f} d \Omega+\int_{\Omega_{f}} \rho_{f}^{-1} \nabla q \cdot \nabla w_{f} d \Omega \\
& -\int_{\Gamma_{f}} \rho_{f}^{-1} c_{F}^{-1} \frac{\partial q}{\partial t} w_{f}-\int_{\Gamma_{i}} \frac{\partial \mathbf{v}}{\partial t} \cdot \mathbf{n}_{\mathbf{s}} w_{f} d \Gamma=\int_{\Omega_{f}} f w_{f} d \Omega,
\end{aligned}
$$

for all test functions $\mathbf{w}_{\mathbf{s}} \in W_{s}$ and $w_{f} \in W_{f}$, where the Sobolev trial spaces $V$ and $Q$ are defined as

$$
\begin{aligned}
& V=\left\{\mathbf{v} \in \mathcal{H}^{1}\left(\Omega_{s}\right)\right\}, \\
& Q=\left\{q \in \mathcal{H}^{1}\left(\Omega_{f}\right): q=0 \text { on } \Gamma_{t}\right\},
\end{aligned}
$$

whereas the test spaces $W_{s}$ and $W_{f}$ are defined as

$$
\begin{aligned}
& W_{s}=\left\{\mathbf{w}_{\mathbf{s}} \in \mathcal{H}^{1}\left(\Omega_{s}\right): \mathbf{w}_{\mathbf{s}}=0 \text { on } \Gamma_{s}\right\}, \\
& W_{f}=\left\{w_{f} \in \mathcal{H}^{1}\left(\Omega_{f}\right): w_{f}=0 \text { on } \Gamma_{f}\right\} .
\end{aligned}
$$

\section{The new non-conforming technique}

Using conforming meshes, i.e. when a vertex of the solid mesh on the interface $\Gamma_{i}$ is also a vertex of the fluid mesh and conversely, there is no particular difficulty to discretize the above variational formulation. However, since the physical parameters, and in particular the wave speeds, may differ strongly in the two subdomains, it is often necessary to use very different space steps in the fluid and in the solid and, consequently, non-conforming meshes at the interface $\Gamma_{i}$.

Before introducing our new non-conforming coupling strategy at the interface $\Gamma_{i}$, let us first explain with the aid of Fig. 2 the essential drawbacks faced when considering a naive approach for a non-conforming coupling in Finite Element Methods. In Fig. 2, the dotted green line represents the actual geometrical interface of problem (1), $\Gamma_{i}$, which must be matched as accurate as possible by the finite element mesh. 


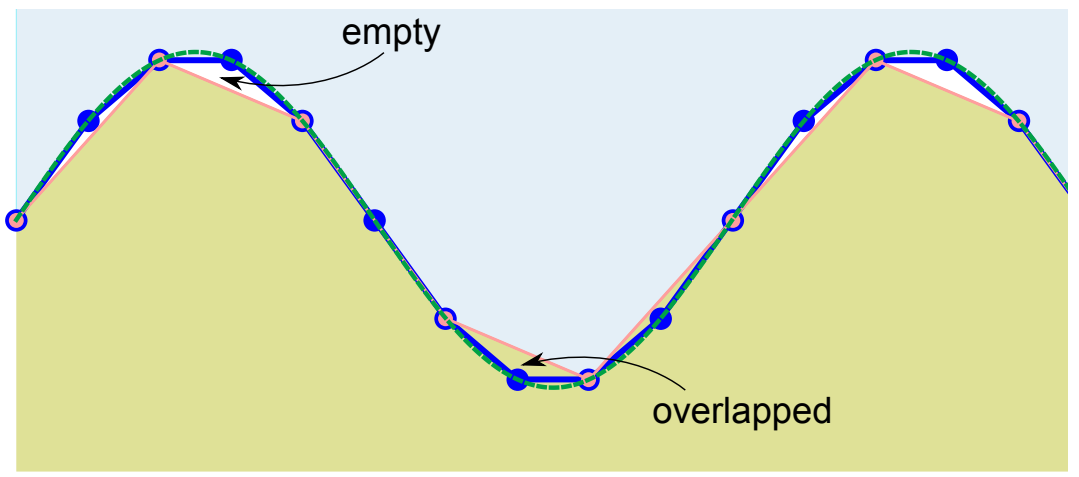

Figure 2: Drawbacks produced when considering a naive non-conforming coupling due to the presence of both empty and overlapped regions of the domain. The green dotted line represents the actual geometry of the interface, $\Gamma_{i}$. The blue and red lines represent the approximation of the geometry from the fluid and the solid side, respectively.

The first step to discretize both subdomains consists in introducing a triangulation $\mathcal{T}_{h_{k}}$ made up of non-overlapping elements within each domain, whose characteristic linear size is denoted by $h_{k}$, and such that $\Omega_{f}=\bigcup_{i=1}^{I_{k}} k_{f}^{i}$ and $\Omega_{s}=\bigcup_{j=1}^{J_{k}} k_{s}^{j}$. Note that this independent decomposition may geometrically introduce a non-conforming coupling between the two subdomains. To understand the root of this issue, let first consider the use of linear elements whose sides are straight lines, commonly referred to as $P 1-$ elements. In each subdomain, the set of edges whose end points belong to the interface $\Gamma_{i}$ determine a particular discretization $\Gamma_{i h}$, say $\Gamma_{i h}^{1}$ for the fluid interface, and another one, $\Gamma_{i h}^{2}$ for the solid interface, identified in Fig. 2 as the blue and the red line, respectively. As it is clear from the figure, empty and overlapped regions are typically generated in this approach when modeling a curved interface, corresponding to the green dotted line. This strategy will therefore introduce numerical instabilities and must be avoided. The source of this problem comes from the fact that two independent, non-matching polygons are used in order to match the nodes projected on $\Gamma_{i}$, say $\Gamma_{i}^{\text {polygon } 1}$ and $\Gamma_{i}^{\text {polygon } 2}$, as represented in the following diagram

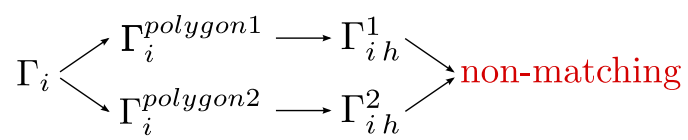

To avoid this problem, we propose a non-conforming solution built on following ingredients: the empty and overlapped regions produced by the naive approach just described must be avoided without adding a significant extra computational cost and, at the same time, the accuracy of the geometry must be of the same order as that of the discretization in space, $p$.

Let first start with $p=1$ with the aid of Fig. 3. Again, the dotted green line represents the actual geometrical interface of problem (1), $\Gamma_{i}$. Now, we consider a unique polygon interpolant on the interface $\Gamma_{i}$, defined such that it interpolates over the set of nodes 


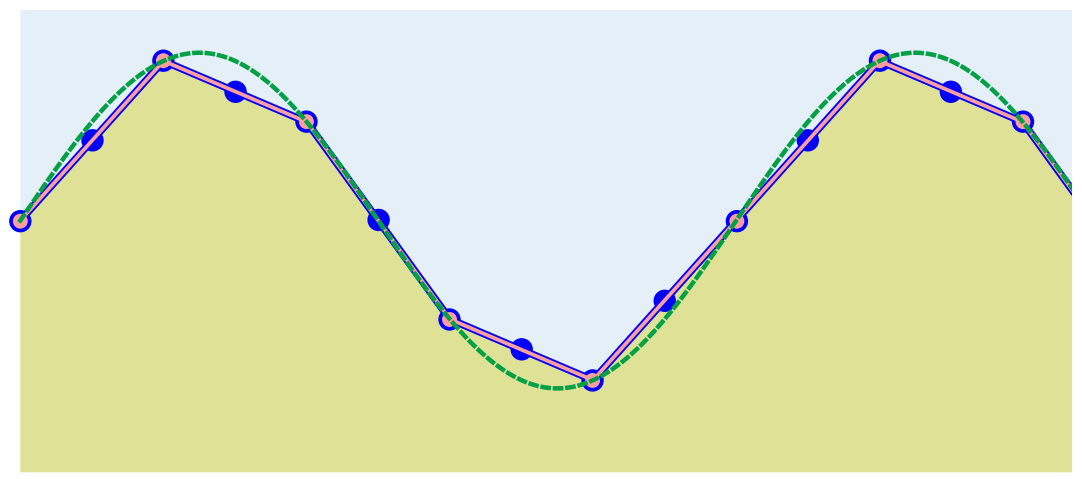

Figure 3: Non-conforming interface matching through spline interpolation of order 1 (polygon). The green dotted line represents the actual geometry of the interface, $\Gamma_{i}$. The blue and red lines represent the approximation of the geometry from the fluid and the solid side, respectively.

that belongs to that subdomain with a coarser mesh refinement requirement. To make these ideas more concrete, let us assume that this is the case of the solid subdomain. 135 Then, from the solid part, we construct a polygon $\Gamma_{i}^{\text {polygon }}$ that interpolates over the set of nodes of the solid mesh that belongs to the interface, building $\Gamma_{i h}^{2}$ (in red). Next, we project over $\Gamma_{i}^{\text {polygon }}$ the nodes of the fluid part that belongs to the interface, building $\Gamma_{i h}^{1}$ (in blue). At this stage, all nodes from both sides belong to a unique polygon and therefore - with the condition that the nodes of the polygon are also vertices in both $\Gamma_{i h}^{1}$ ${ }_{140}$ and $\Gamma_{i h}^{2}$-we ensure that $\Gamma_{i h}^{1}$ and $\Gamma_{i h}^{2}$ are now matching. This idea is represented in the following diagram

$$
\Gamma_{i} \longrightarrow \Gamma_{i}^{\text {polygon }} \searrow \begin{aligned}
& \Gamma_{i h}^{1} \\
& \Gamma_{i h}^{2}
\end{aligned} \searrow^{\text {matching }}
$$

The same idea can now be extended to higher order, $p>1$, using a spline interpolant of order $r \leq p$, spline $(r)$. For the sake of simplicity we consider $r=p$. As in the $P 1$ case, once all nodes that belongs to the interface have been placed following our strategy, we proceed to build up the two meshes whose elements are of order $p$. Keeping all these aspects in consideration, the resulting meshes from the fluid and the solid side are approximating the geometry of $\Gamma_{i}$ with order $p$. Additionally, $\Gamma_{i h}^{1}=\Gamma_{i h}^{2}$ provided that a knot that belongs to $\operatorname{spline}(p)$ is also a vertex in both $\Gamma_{i h}^{1}$ and $\Gamma_{i h}^{2}$. Again, this idea can be represented in the next diagram

$$
\Gamma_{i} \longrightarrow \Gamma_{i}^{\text {spline }(r)} \ \begin{aligned}
& \Gamma_{i h}^{1} \\
& \Gamma_{i h}^{2}
\end{aligned} \nearrow_{\text {matching }}
$$

150

From now on we may refer to $\Gamma_{i h}^{1}=\Gamma_{i h}^{2}$ simply as $\Gamma_{i h}$. The interface $\Gamma_{i h}$ can be compared to the glue mesh proposed in [31] for SBP finite differences methods. In Figs. 4 
and 5 , we provide an illustration of these ideas for the particular case of $p=2$. There, the nodes from the fluid and solid side match perfectly on the interface which is defined by a common spline of order $p$. We also remark how the accuracy of the actual geometry improves with increasing order $p$, in consonance with the order of approximation used in the spatial discretization.

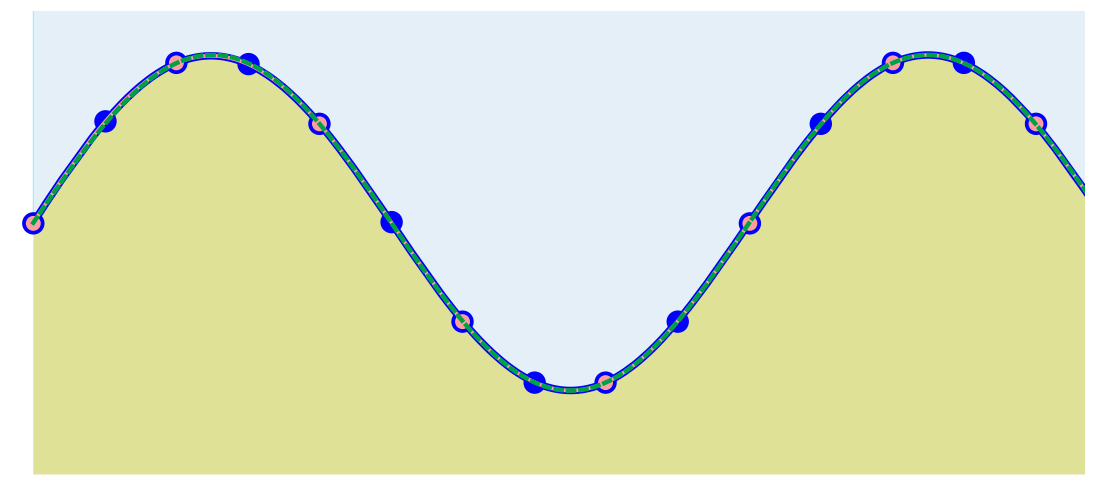

Figure 4: Non-conforming interface matching through spline interpolation of order 2. The green dotted curve represents the actual geometry of the interface, $\Gamma_{i}$. The blue and red lines represent the approximation of the geometry from the fluid and the solid side, respectively.

Two illustrations of a mesh produced by our non-conforming technique is shown in Fig. 6 for $P 1$ - and $P 2$-elements. Note here that between two given nodes of the spline that builds $\Gamma_{i h}$, they could be an arbitrary number of elements that belongs to both $\Gamma_{i h}^{1}$ and $160 \Gamma_{i h}^{2}$, independent of each other. Consequently, as an advantage in our approach, there is no specific pattern that geometrically constrains the relation of refinement between $\Gamma_{i h}^{1}$ and $\Gamma_{i h}^{2}$.

In the next section we explain the space discretization used and how to deal properly with the numerical integration over $\Gamma_{i h}$.

\section{Discretization in space and algorithmic treatment of the non-conforming coupling}

We now introduce the finite-dimensional trial spaces as $Q_{h} \subset Q$, and $V_{h} \subset V$, and test spaces as $W_{s_{h}} \subset W_{s}$, and $W_{f_{h}} \subset W_{f}$. For the sake of simplicity we introduce the following notation

$$
\mathbf{u}:=\mathbf{u}_{\mathbf{h}}=\left(q, v_{1}, v_{2}\right)^{T},
$$

where

$$
\begin{array}{ll}
q:=q_{h}=\sum_{j=1}^{N_{f}} q_{j} N_{f, j}, & F=(f, 0,0)^{T}, \\
v_{1}:=v_{1_{h}}=\sum_{j=1}^{N_{s}} v_{1, j} N_{s, j}, & v_{2}:=v_{2_{h}}=\sum_{j=1}^{N_{s}} v_{2, j} N_{s, j},
\end{array}
$$




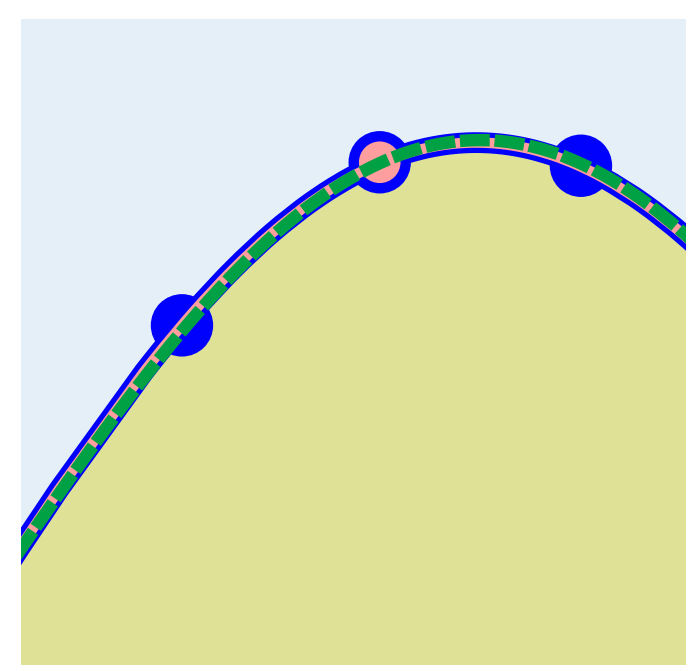

Figure 5: Detail of the non-conforming interface: The blue and red curves (fluid and solid interface) are exactly the same, produced by a common spline interpolation. Both curves approach the actual geometry (green dotted curve) of the interface $\Gamma_{i}$ with order 2.

and $N_{f}$ and $2 N_{s}$ represent the number of degrees of freedom in the fluid and the solid part, respectively. The matrix system associated to problem (5) is defined as

$$
M \frac{\partial^{2} \mathbf{u}}{\partial t^{2}}+(S+C) \frac{\partial \mathbf{u}}{\partial t}+K u=F,
$$

whose block matrices of size $2 N_{s} N_{f} \times 2 N_{s} N_{f}$ are defined as

$$
\begin{array}{rlrl}
M= & \left(\begin{array}{ccc}
M_{f} & 0 & 0 \\
0 & M_{s 1} & 0 \\
0 & 0 & M_{s 2}
\end{array}\right), & S=\left(\begin{array}{ccc}
S_{f} & 0 & 0 \\
0 & S_{s 11} & S_{s 12} \\
0 & S_{s 21} & S_{s 22}
\end{array}\right), \\
C=\left(\begin{array}{ccc}
0 & C_{f, s 1} & C_{f, s 2} \\
C_{s 1, f} & 0 & 0 \\
C_{s 2, f} & 0 & 0
\end{array}\right), & K=\left(\begin{array}{ccc}
K_{f} & 0 & 0 \\
0 & K_{s 11} & K_{s 12} \\
0 & K_{s 21} & K_{s 22}
\end{array}\right) .
\end{array}
$$

Regarding the fluid equations, the mass $(M)$, stiffness $(F)$ and absorbing $(S)$ matrices and vector entries are given component-wise as

$$
\begin{array}{ll}
\left(M_{f}\right)_{i j}=\int_{\Omega_{f h}} \rho_{f}^{-1} c_{F}^{-2} N_{f, i} N_{f, j} d \Omega, & \left(S_{f}\right)_{i j}=\int_{\Gamma_{f h}} \rho_{f}^{-1} c_{F}^{-1} N_{f, i} N_{f, j} d \Gamma, \\
\left(K_{f}\right)_{i j}=\int_{\Omega_{f h}} \rho_{f}^{-1}\left(\frac{\partial N_{f, i}}{\partial x_{1}} \frac{\partial N_{f, j}}{\partial x_{1}}+\frac{\partial N_{f, i}}{\partial x_{2}} \frac{\partial N_{f, j}}{\partial x_{2}}\right) d \Omega, & (F)_{i}=\int_{\Omega_{f h}} f N_{f, i} d \Omega,
\end{array}
$$




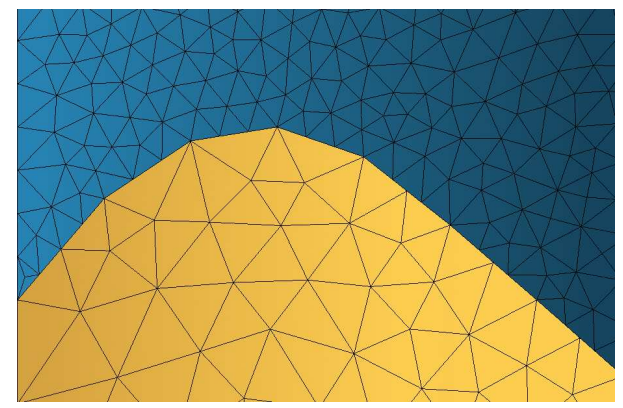

(a) P1-elements

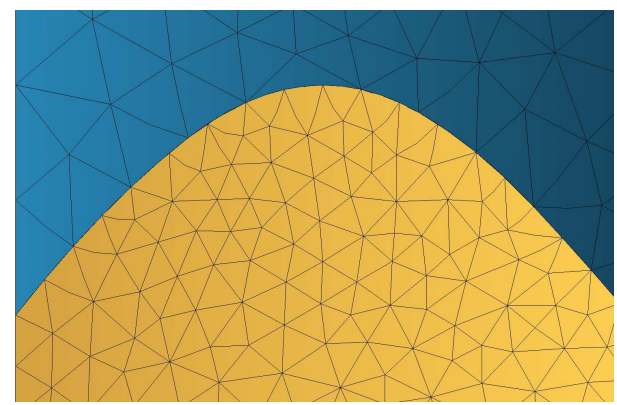

(b) P2-elements

Figure 6: Illustration of a non-conforming mesh produced by our technique for linear and curved elements, zooming in on the coupling part. No specific pattern of refinement between the two domains is imposed.

whereas for the solid equations, as

$$
\begin{array}{ll}
\left(M_{s 11}\right)_{i j}=\int_{\Omega_{s h}} \rho_{s} N_{s, i} N_{s, j} d \Omega, & \left(M_{s 22}\right)_{i j}=\int_{\Omega_{s h}} \rho_{s} N_{s, i} N_{s, j} d \Omega, \\
\left(S_{s 11}\right)_{i j}=\int_{\Gamma_{s h}} \rho_{s}\left(c_{P} n_{s 1}^{2}+c_{S} n_{s 2}^{2}\right) N_{s, i} N_{s, j} d \Gamma, \quad\left(S_{s 12}\right)_{i j}=\int_{\Gamma_{s h}} \rho_{s}\left(c_{P}-c_{S}\right) n_{s 1} n_{s 2} N_{s, i} N_{s, j} d \Gamma, \\
\left(S_{s 21}\right)_{i j}=\int_{\Gamma_{s h}} \rho_{s}\left(c_{P}-c_{S}\right) n_{s 1} n_{s 2} N_{s, i} N_{s, j} d \Gamma, \quad\left(S_{s 22}\right)_{i j}=\int_{\Gamma_{s h}} \rho_{s}\left(c_{P} n_{s 2}^{2}+c_{S} n_{s 1}^{2}\right) N_{s, i} N_{s, j} d \Gamma, \\
\left(K_{s 11}\right)_{i j}=\int_{\Omega_{s h}} \lambda \frac{\partial N_{s, i}}{\partial x_{1}} \frac{\partial N_{s, j}}{\partial x_{1}}+2 \mu\left(\frac{\partial N_{s, i}}{\partial x_{1}} \frac{\partial N_{s, j}}{\partial x_{1}}+\frac{1}{2} \frac{\partial N_{s, i}}{\partial x_{2}} \frac{\partial N_{s, j}}{\partial x_{2}}\right) d \Omega, \\
\left(K_{s 12}\right)_{i j}=\int_{\Omega_{s h}} \lambda \frac{\partial N_{s, i}}{\partial x_{1}} \frac{\partial N_{s, j}}{\partial x_{2}}+\mu \frac{\partial N_{s, i}}{\partial x_{2}} \frac{\partial N_{s, j}}{\partial x_{1}} d \Omega, \\
\left(K_{s 21}\right)_{i j}=\int_{\Omega_{s h}} \lambda \frac{\partial N_{s, i}}{\partial x_{2}} \frac{\partial N_{s, j}}{\partial x_{1}}+\mu \frac{\partial N_{s, i}}{\partial x_{1}} \frac{\partial N_{s, j}}{\partial x_{2}} d \Omega, \\
\left(K_{s 22}\right)_{i j}=\int_{\Omega_{s h}} \lambda \frac{\partial N_{s, i}}{\partial x_{2}} \frac{\partial N_{s, j}}{\partial x_{2}}+2 \mu\left(\frac{1}{2} \frac{\partial N_{s, i}}{\partial x_{1}} \frac{\partial N_{s, j}}{\partial x_{1}}+\frac{\partial N_{s, i}}{\partial x_{2}} \frac{\partial N_{s, j}}{\partial x_{2}}\right) d \Omega .
\end{array}
$$

We use a standard treatment to compute the numerical quadrature, but it useful to detail how we overcome the technical difficulties related to our non-conforming coupling technique. These integrals appear in the coupling matrices $(C)$ and are given componentwise as follows

$$
\begin{aligned}
\left(C_{f s 1}\right)_{i j} & =-\int_{\Gamma_{i h}} n_{s 1} N_{f, i} N_{s, j} d \Gamma, & \left(C_{f s 2}\right)_{i j} & =-\int_{\Gamma_{i h}} n_{s 2} N_{f, i} N_{s, j} d \Gamma, \\
\left(C_{s 1 f}\right)_{i j} & =-\int_{\Gamma_{i h}} n_{f 1} N_{s, i} N_{f, j} d \Gamma, & \left(C_{s 2 f}\right)_{i j} & =-\int_{\Gamma_{i h}} n_{f 1} N_{s, i} N_{f, j} d \Gamma .
\end{aligned}
$$

As shown in Fig. 7, from the algorithmic point of view there are four special cases that must be considered in order to determine properly the limits of integration performed over a given intersection of two given edges (shown in red). Let us consider that along $170 \quad \Gamma_{i h}$, there are $n_{f}$ edges from the fluid side and $n_{s}$ edges from the solid side. Let $e_{l}^{f}$ and 


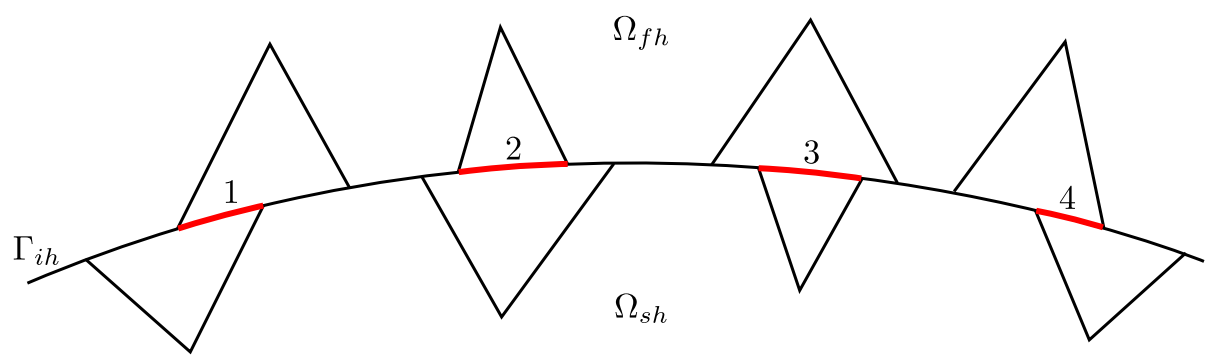

Figure 7: The four possible scenarios to deal with the numerical quadrature in our non-conforming technique.

$e_{m}^{s}$ denote respectively, a particular edge from the fluid and the solid along $\Gamma_{i h}$, so that $\Gamma_{i h}=\cup_{l=1}^{n_{f}} e_{l}^{f}=\cup_{m=1}^{n_{s}} e_{m}^{s}$. Obviously $\Gamma_{i h}=\cup_{l=1}^{n_{f}} \cup_{m=1}^{n_{s}}\left(e_{l}^{f} \cap e_{m}^{s}\right)$. To fix the ideas, let us consider without loss of generality the following representative integral from (12),

$$
\int_{e_{l}^{f} \cap e_{m}^{s}} N_{f, a} N_{s, b} d \Gamma
$$

over the curved determined by $e_{l}^{f} \cap e_{m}^{s}$, as illustrated in red in Fig. 8 (corresponding to the fourth case in Fig. 7). We denote by $k_{1}^{f}$ the fluid element whose interfacial edge is $e_{l}^{f}$ and by $k_{2}^{s}$ the solid element whose interfacial edge is $e_{m}^{s}$.

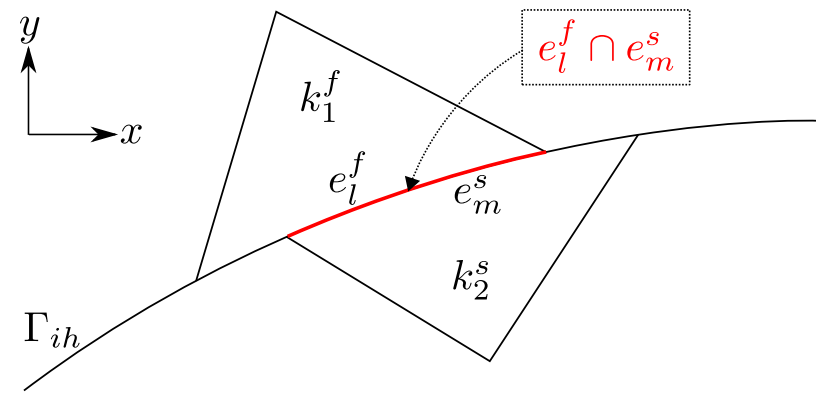

Figure 8: Illustration of the numerical quadrature performed over the intersection of two edges, $e_{l}^{f} \cap e_{m}^{s}$, where $e_{l}^{f} \in k_{1}^{f}$ and $e_{m}^{s} \in k_{2}^{s}$.

Let us for instance assume that we compute the integral "from the fluid side", i.e. by using the map $F_{k_{1}^{f}}$ that transforms the reference element $\hat{k}_{1}^{f}$ into the fluid element $k_{1}^{f}$. The difficulty in evaluating such integral comes from the fact that, while the shape function $N_{f, a}$ can be easily evaluated, the evaluation of the shape function $N_{s, b}$ from the solid side is much more tedious. To illustrate this, it is necessary to introduce additional notation related to the parametric space. Regarding the coordinates, we define a (non-linear) map $\mathbf{x}=(x(\xi, \eta), y(\xi, \eta))=F_{k_{1}^{f}}(\xi, \eta)$ from the parametric space in $\hat{k}$-with $\xi \in[0,1]$ 
and $\eta \in[0,1-\xi]$ — to the physical space in $k_{1}^{f}$, given by

$$
F_{k_{1}^{f}}(\xi, \eta)=\sum_{i=1}^{n_{k_{1}^{f}}} \mathbf{x}_{k_{1}^{f}, i} \hat{N}_{f, i}(\xi, \eta),
$$

where $n_{k_{1}^{f}}$ is the number of Lagrange shape functions defined on $k_{1}^{f}$ which obviously depends on $p, \hat{N}_{f, i}$ is the $i$-th Lagrange shape function of degree $p$ on the reference element $\hat{k}_{1}^{f}$ and $\mathbf{x}_{k_{1}^{f}, i}$ represents the physical coordinate of the degree of freedom associated to the $i$-th Lagrange shape function. Since our integral is performed over a particular edge of $k_{1}^{f}, e_{l}^{f}$ (specifically, over the intersection with $e_{m}^{s}$ ), we introduce a bijective parameterization $\mathbf{r}_{e_{l}}^{f}:[0,1] \rightarrow e_{l}^{f}$ of $e_{l}^{f}$. The function can be easily constructed with the aid of an auxiliary function $\left(\xi_{\hat{e}_{k_{1}}^{f}}\left(t^{f}\right), \eta_{\hat{e}_{k_{1}}^{f}}\left(t^{f}\right)\right)=\boldsymbol{\rho}\left(t^{f}\right)$ such that $\boldsymbol{\rho}\left(t^{f}\right)=\left(t^{f}, 0\right)$, $\boldsymbol{\rho}\left(t^{f}\right)=\left(1-t^{f}, t^{f}\right)$ or $\boldsymbol{\rho}\left(t^{f}\right)=\left(0,1-t^{f}\right)$, depending on the edge that we are considering. Then, $\mathbf{r}_{e_{l}}^{f}\left(t^{f}\right)=F_{k_{1}^{f}}\left(\boldsymbol{\rho}\left(t^{f}\right)\right)$ and

$$
\mathbf{r}_{e_{l}}^{f}\left(t^{f}\right)=\sum_{i=1}^{n_{k_{1}^{f}}} \mathbf{x}_{k_{1}^{f}, i} \hat{N}_{f, i}\left(\xi_{\hat{e}_{l}^{f}}\left(t^{f}\right), \eta_{\hat{e}_{l}^{f}}\left(t^{f}\right)\right) .
$$

Similarly in the solid side, a bijective parameterization of $e_{m}^{s}$ can be written as

$$
\mathbf{r}_{e_{m}}^{s}\left(t^{s}\right)=\sum_{i=1}^{n_{k_{2}^{s}}} \mathbf{x}_{k_{2}^{s}, i} \hat{N}_{s, i}\left(\xi_{\hat{e}_{m}^{s}}\left(t^{s}\right), \eta_{\hat{e}_{m}^{s}}\left(t^{s}\right)\right) .
$$

Now, let $t_{0}^{f}$ and $t_{1}^{f}$ be such that $\mathbf{r}_{e_{l}}^{f}\left(t_{0}^{f}\right)$ and $\mathbf{r}_{e_{l}}^{f}\left(t_{1}^{f}\right)$ give the physical endpoints of $e_{l}^{f} \cap e_{m}^{s}, 0 \leq t_{0}^{f} \leq t_{1}^{f} \leq 1$.

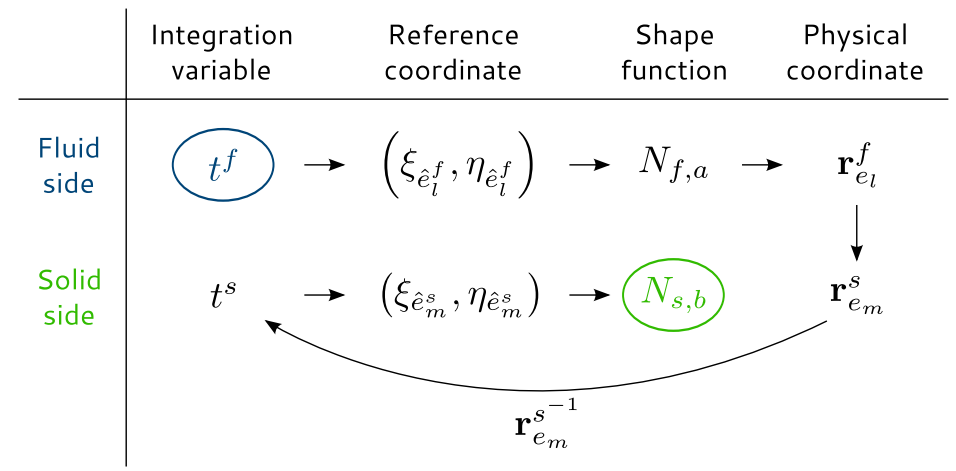

Figure 9: Diagram of the evaluation of the shape function $N_{s, b}$ in the solid side, starting from the variable of integration $t^{f}$ in the fluid side. Every arrow connecting two terms implies that the second one may be obtained from the first one. A term over the arrow specifies how the second term is actually obtained from the first as an argument.

With this notation, note that the numerical integration in (13) is performed in the fluid side with a integration variable $t^{f}$ between end points $t_{0}^{f}$ and $t_{1}^{f}$. The evaluation of 
$N_{s, b}$ from $t^{f}$ is schematically summarized in Fig.9. Note that the connection between the fluid side and the solid side is possible in our approach because of the perfect matching between the two sides on $\Gamma_{i}$. In fact,

$$
\text { if } e_{l}^{f} \cap e_{m}^{s} \neq \varnothing \Rightarrow \forall t^{f} \in\left[t_{0}^{f}, t_{1}^{f}\right] \exists t^{s} \in[0,1] \mid \mathbf{r}_{e_{l}}^{f}\left(t^{f}\right)=\mathbf{r}_{e_{m}}^{s}\left(t^{s}\right) .
$$

Notice, however, that we cannot in general compute the inverse of the map $\mathbf{r}(t)$ if we consider curvilinear elements. To solve this final issue, we need to consider that, within an element, the map $\mathbf{r}(t)$ is monotonically increasing in either $x$ - or $y$-direction. If it is the case, say for $x$, we are sure that we can numerically determine the root of $x-\mathbf{r}^{-1}(t)_{x}$, as it has multiplicity $p$ and is therefore unique. To find such a root numerically within a fixed tolerance, a bisection method may be used with linear convergence.

With the above notation, the representative integral in (13) may be then written in the reference edge $\hat{e}_{l}^{f}$ as

$$
\begin{aligned}
& \int_{t_{0}^{f}}^{t_{1}^{f}} d t^{f} N_{f, a}\left(\xi_{\hat{e}_{l}^{f}}\left(t^{f}\right), \eta_{\hat{e}_{l}^{f}}\left(t^{f}\right)\right) \\
& \times N_{s, b}\left(\xi_{\hat{e}_{m}^{s}}\left(\mathbf{r}_{e_{m}}^{s^{-1}}\left(\mathbf{r}_{e_{l}}^{f}\left(t^{f}\right)\right)\right), \eta_{\hat{e}_{m}^{s}}\left(\mathbf{r}_{e_{m}}^{s^{-1}}\left(\mathbf{r}_{e_{l}}^{f}\left(t^{f}\right)\right)\right)\right) \\
& \times\left(\left(\partial x / \partial t^{f}\right)^{2}+\left(\partial y / \partial t^{f}\right)^{2}\right)^{1 / 2},
\end{aligned}
$$

where

$$
\mathbf{r}_{e_{m}}^{s^{-1}}\left(\mathbf{r}_{e_{l}}^{f}\left(t^{f}\right)\right)=t^{s}
$$

and

$$
(x, y)=\mathbf{r}_{e_{l}}^{f}\left(t^{f}\right)
$$

\section{Discretization in time and stability analysis}

Regarding the stability analysis we consider the absence of the source term for convenience, i.e., $F=0$. At this stage, we would like to point out that the family of Runge-Kutta time-discretization schemes may well deserve attention in a future work. In this paper we stick to the well-known Leap-frog scheme for the discretization in time of the second derivative, whereas for the first derivative we apply central differences, to obtain the following second-order explicit scheme

$$
\begin{aligned}
& M_{f} \frac{q^{n+1}-2 q^{n}+q^{n-1}}{\Delta t^{2}}+S_{f} \frac{q^{n+1}-q^{n-1}}{2 \Delta t}+C_{f s} \frac{v^{n+1}-v^{n-1}}{2 \Delta t}+K_{f} q^{n}=0 . \\
& M_{s} \frac{v^{n+1}-2 v^{n}+v^{n-1}}{\Delta t^{2}}+S_{s} \frac{v^{n+1}-v^{n-1}}{2 \Delta t}+C_{s f} \frac{q^{n+1}-q^{n-1}}{2 \Delta t}+K_{s} v^{n}=0,
\end{aligned}
$$

Multiplying equations (15) and (16) by $\frac{q^{n+1}-q^{n-1}}{2 \Delta t}$ and $\frac{v^{n+1}-v^{n-1}}{2 \Delta t}$, respectively, summing them up and using

$$
u^{n+1}-2 u^{n}+u^{n-1}=\left(u^{n+1}-u^{n}\right)-\left(u^{n}-u^{n-1}\right)
$$


and

$$
u^{n+1}-u^{n-1}=\left(u^{n+1}-u^{n}\right)+\left(u^{n}-u^{n-1}\right),
$$

we finally obtain the following expression

$$
\begin{aligned}
& \frac{1}{2 \Delta t}<M_{s} \frac{v^{n+1}-v^{n}}{\Delta t}, \frac{v^{n+1}-v^{n}}{\Delta t}>-<M_{s} \frac{v^{n}-v^{n-1}}{\Delta t}, \frac{v^{n}-v^{n-1}}{\Delta t}> \\
& +<S_{s} \frac{v^{n+1}-v^{n-1}}{2 \Delta t}, \frac{v^{n+1}-v^{n-1}}{2 \Delta t}>+\frac{<K_{s} v^{n+1}, v^{n}>-<K_{s} v^{n}, v^{n-1}>}{2 \Delta t} \\
& +\frac{1}{2 \Delta t}<M_{f} \frac{q^{n+1}-q^{n}}{\Delta t}, \frac{q^{n+1}-q^{n}}{\Delta t}>-<M_{f} \frac{q^{n}-q^{n-1}}{\Delta t}, \frac{q^{n}-q^{n-1}}{\Delta t}> \\
& +<S_{f} \frac{q^{n+1}-q^{n-1}}{2 \Delta t}, \frac{q^{n+1}-q^{n-1}}{2 \Delta t}>+\frac{<K_{f} q^{n+1}, q^{n}>-<K_{f} q^{n}, q^{n-1}>}{2 \Delta t} \\
& =0 .
\end{aligned}
$$

Now, a discrete energy can be introduced for $v$ (and similarly for $q$ ) as

$$
E_{v}^{i+1}=\frac{1}{2}\left\|\frac{v^{n+1}-v^{n}}{\Delta t}\right\|^{2}+\frac{1}{2}\left\langle M_{s}^{-1} K_{s} v^{n+1}, v^{n}\right\rangle,
$$

where the following condition holds (in absence of the source $F$, which vanishes after a given time)

$$
\begin{aligned}
\frac{1}{\Delta t}\left(E_{v}^{i+1}-E_{v}^{i}\right)+\frac{1}{\Delta t}\left(E_{q}^{i+1}-E_{q}^{i}\right)= & -<M_{s}^{-1} S_{s} \frac{v^{n}-v^{n-1}}{\Delta t}, \frac{v^{n}-v^{n-1}}{\Delta t}> \\
& -<M_{f}^{-1} S_{f} \frac{q^{n}-q^{n-1}}{\Delta t}, \frac{q^{n}-q^{n-1}}{\Delta t}>,
\end{aligned}
$$

which shows, using the positivity of matrices $M_{f}, M_{s}, S_{f}$ and $S_{s}$, that the discrete energy decreases in time.

However, the term $\left\langle M_{s}^{-1} K_{s} v^{n+1}, v^{n}\right\rangle$ present in (18) does not guarantee the positivity of the discrete energy. Nevertheless, we first notice that

$$
\begin{aligned}
& \frac{1}{2}\left\|\frac{v^{n+1}-v^{n}}{\Delta t}\right\|^{2} \\
& +\frac{1}{2}\left(\left\langle M_{s}^{-1} K \frac{v^{n+1}+v^{n}}{2}, \frac{v^{n+1}+v^{n}}{2}\right\rangle-\frac{\Delta t^{2}}{4}\left\langle M_{s}^{-1} K_{s} \frac{v^{n+1}-v^{n}}{\Delta t}, \frac{v^{n+1}-v^{n}}{\Delta t}\right\rangle\right) \\
& =0 .
\end{aligned}
$$

Defining

$$
\left\|A_{s}\right\|=\sup _{v} \frac{\left\langle I_{s}-M_{s}^{-1} K_{s} v, v\right\rangle}{\|v\|^{2}}
$$

it holds that

$$
\left\langle I_{s}-M_{s}^{-1} K_{s} \frac{v^{n+1}-v^{n}}{\Delta t}, \frac{v^{n+1}-v^{n}}{\Delta t}\right\rangle \leq\left\|A_{s}\right\|\left\|\frac{v^{n+1}-v^{n}}{\Delta t}\right\|^{2},
$$


and then, the discrete energy satisfies

$$
E_{v}^{i+1} \geq\left(I_{s}-\frac{\Delta t^{2}}{4}\left\|A_{s}\right\|\right)\left\|\frac{v^{n+1}-v^{n}}{\Delta t}\right\|^{2}+\left\langle M_{s}^{-1} K_{s} \frac{v^{n+1}+v^{n}}{2}, \frac{v^{n+1}+v^{n}}{2}\right\rangle .
$$

A sufficient condition that ensures the positivity of $E_{v}^{i+1}$ is therefore

$$
\Delta t^{2}<\frac{4}{\left\|A_{s}\right\|} .
$$

In order to go deeper in our analysis, we consider now the case of rectangular subdomains with a regular mesh consisting of triangles with straight sides where square elements are first divided into two triangles. In this setting, the largest eigenvalue of $\left\|A_{s}\right\|$ behaves as $\alpha_{s} / h_{s}^{2}, \alpha_{s}$ depending only on the space discretization method, and so

$$
\Delta t<\frac{2 h_{s}}{\sqrt{\alpha_{s} d}}
$$

where $d$ is the dimension of the domain $\Omega$. Similarly for $q$, we obtain

$$
\Delta t<\frac{2 h_{f}}{\sqrt{\alpha_{f} d}}
$$

and thus the final CFL condition for our coupled problem reads

$$
\Delta t<\min \left(\frac{2 h_{f}}{\sqrt{\alpha_{f} d}}, \frac{2 h_{s}}{\sqrt{\alpha_{s} d}}\right) .
$$

This shows that the coupling does not penalize the CFL condition.

On the other hand, considering the stiffness terms implicitly, i.e.

$$
K_{f} q(n \Delta t) \approx \frac{1}{2} K_{f}\left(q^{n+1}+q^{n-1}\right)
$$

and

$$
K_{s} v(n \Delta t) \approx \frac{1}{2} K_{s}\left(v^{n+1}+v^{n-1}\right)
$$

in (15) and (16), similar calculations lead us to an unconditional stable and conservative scheme where the discrete energy satisfies:

$$
E_{v}^{i+1}=\frac{1}{2}\left\|\frac{v^{n+1}-v^{n}}{\Delta t}\right\|^{2}+\frac{1}{2}\left\langle M_{s}^{-1} K_{s} v^{n+1}, v^{n+1}\right\rangle+\frac{1}{2}\left\langle M_{s}^{-1} K_{s} v^{n}, v^{n}\right\rangle .
$$

190

Notice that this implicit scheme does not introduce any additional computational cost with respect to the explicit scheme, as it also involves only one matrix factorization operation. Moreover, the sparsity structure of the matrix to be inverted remains the same and so does, consequently, the cost of the factorization for a given direct solver used. 


\section{Numerical results}

In this section we first consider a classical reference problem found in ([17]) in order to validate our method, both in terms of accuracy and flexibility. An illustration of the domain of this problem $\Omega=\Omega_{f} \cup \Omega_{s}$ is given in Fig. 10, whose interface $\Gamma_{i}$ is defined by means of a sinusoid. The size of the rectangular domain $\Omega$ is $64 \mathrm{~km} \times 48 \mathrm{~km}$, where the origin of the coordinate system is placed at the lower left corner of the domain.

The equations and boundary conditions imposed are those introduced previously in (1). The parameters in the acoustic domain are given by $\rho_{f}=1020 \mathrm{~kg} \mathrm{~m}^{-3}$, for the density of the homogeneous material, and $c_{F}=1500 \mathrm{~m} \mathrm{~s}^{-1}$ for the $P$-wave speed. In elastic medium, we fix the material density to $\rho_{s}=2500 \mathrm{~kg} \mathrm{~m}^{-3}$, whereas $c_{P}=3400 \mathrm{~m}$

$205 \mathrm{~s}^{-1}$ and $c_{S}=c_{P} / \sqrt{3}$ for the $P$ - and $S$-wave speeds, respectively. The point source time function based on a Ricker wavelet is located in the fluid domain at $\mathbf{x}_{\mathbf{0}}=[29.0833,31]$, with a dominant frequency of $10 \mathrm{~Hz}$. On the other hand, a set of receivers used to register seismograms are placed in both subdomains: in the fluid part, receivers $r_{i}^{f}$ are located at $[32+2.2 i, 33]$, for $i=0, \ldots, 10$; whereas in the solid part, receivers $r_{i}^{s}$ are

210 located at $[32+2.2 i, 15]$, for $i=0, \ldots, 10$. The smallest elements of the computational mesh are located in the fluid side and have a characteristic length about $h=12.5 \mathrm{~m}$, whereas the characteristic length for the elements in the solid side is $h=25 \mathrm{~m}$. As for the discretization in time, the implicit second-order Leap-frog scheme presented in Sec. 6 is used. Regarding the details of implementation, we point out that our code

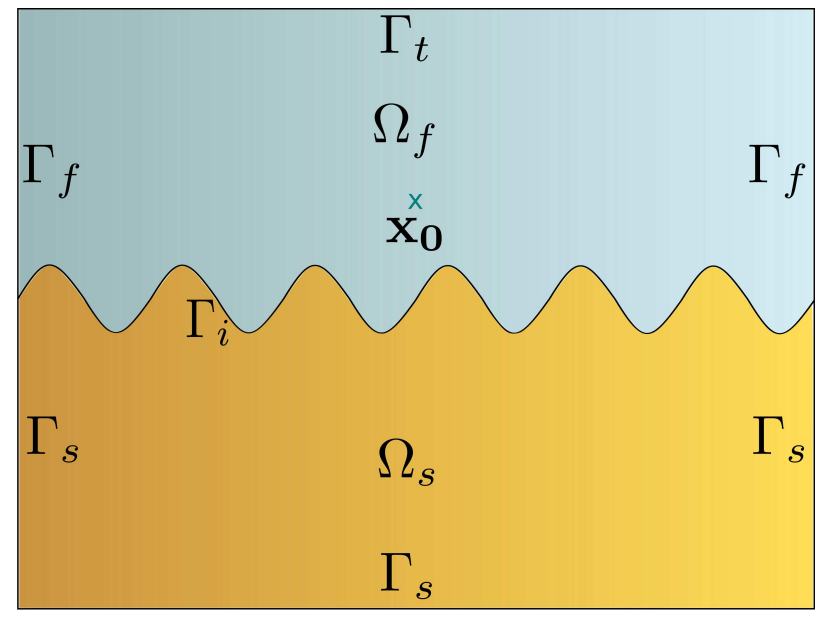

Figure 10: Illustration of the domain of the problem with a sinusoidal interface. The point source located at $\mathbf{x}_{\mathbf{0}}=[29.0833,31]$ is labeled as ' $\times$ '. In fluid part, receivers $r_{i}^{f}$ are located at [32+2.2i,33], whereas in the solid part, at $[32+2.2 i, 15]$, for $i=0, \ldots, 10$.

215 has been completely written in $C / C++$, where the MUMPS [32] package is employed for solving the corresponding linear algebraic system. An important building block that has also been developed consists of a mesh loader for meshes generated by Gmsh [33], suitable for arbitrary high-order triangle elements. In order to compare the results of our method with those provided in [17], where the authors used the Spectral FEM, we show 
220 two snapshots in figures 12 and 13, exactly at the same times, $t=0.87$ and $t=1.57$, respectively. The coincidence of both methods is remarkable and all characteristic waves are identified in both domains as expected. In Fig.11 we show the seismogram registered at receiver $r_{0}^{f}$, located at $[32,15]$. Once the signal emitted from the source passes through it, the rest of the signal registered comes from both the free condition imposed on top of 225 the fluid domain and the numerical reflections due to the first order absorbing boundary conditions imposed. In order to validate further the accuracy of the numerical solutions

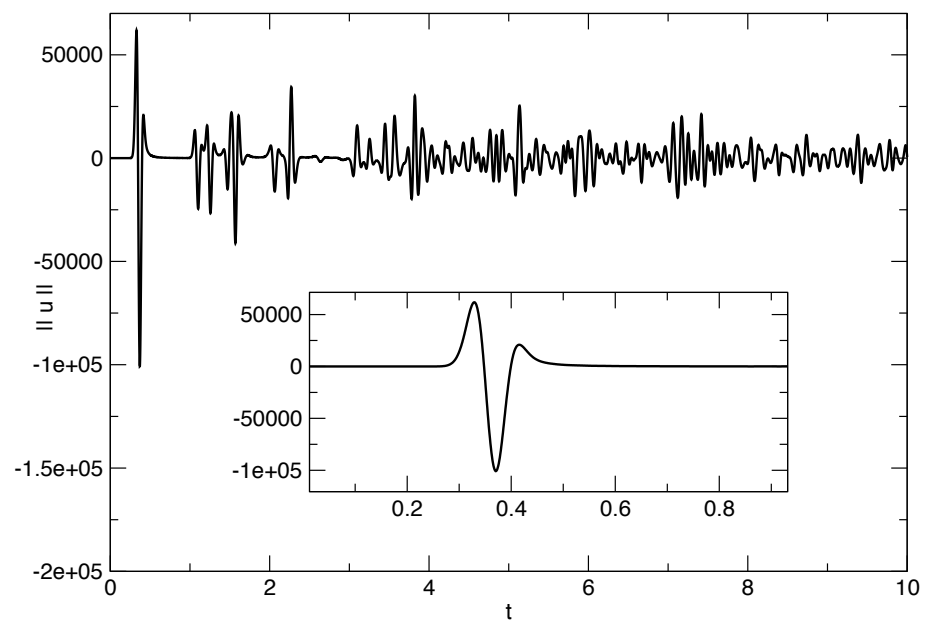

Figure 11: Seismogram recorder at receiver $r_{0}^{f}$. The inner plot represents a zoom from $t=0$ to $t=0.92$, where it is reflected the source signal passing through, without reflections.

provided by our method, we have computed this same seismogram on an extremely fine conforming mesh and used it as a reference solution. In the next table we report numerical errors in the $\mathcal{L}^{2}$-norm obtained when using coarser non-conforming meshes with characteristic length given by $h$, for $p=1$ and $p=2$, achieving the expected rates of convergence.

\begin{tabular}{crr}
\hline \multirow{2}{*}{$h$} & \multicolumn{2}{c}{$\mathcal{L}^{2}-$ error } \\
\cline { 2 - 3 } & $P-1$ & $P-2$ \\
\hline 12.5 & 0.06039971 & 0.000081702 \\
25 & 0.53659014 & 0.004501854 \\
50 & 4.50163058 & 0.342980559 \\
100 & 31.34660553 & 26.12291773 \\
\hline
\end{tabular}




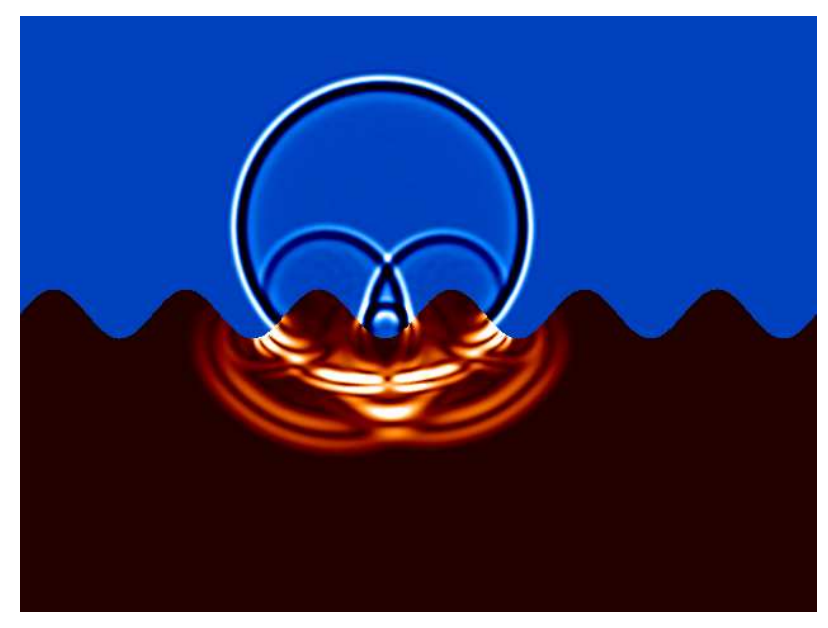

Figure 12: Snapshot of the reference test at time $t=0.87$ secs.

Let us now validate the theoretical convergence of our method. In classical FEM, the following relative $\mathcal{H}^{1}$ error computed along the time

$$
e_{h}:=\frac{\left\|u(\cdot, \cdot)-u_{h}\right\|_{\mathcal{L}^{2}(0, T ; \Omega)}}{\|u(\cdot, \cdot)\|_{\mathcal{L}^{2}(0, T ; \Omega)}}
$$

is dominated by $h^{p+1}$, where $h$ is the characteristic mesh size and $p$ the order of the space discretization. In figures 14 and 15, we show the numerical convergence in log-log scale, achieved by our method for the classical reference test described above, validating the theoretical estimates. In both cases, a very fine numerical solution using a conforming mesh has been computed and considered as a reference solution, since no analytical solution is available for this test problem. Note that the convergence rate reported is slightly better than the theoretical estimates just because the meshes used are unstructured. In the case $p=2$, we observe a regime where the convergence is actually of order $p+1$, as expected. Clearly for $p>1$, for small enough $h$ the dominant error will eventually be associated to the time discretization error of the Leap-frog scheme considered which is of second-order.

Now, we analyze the efficiency of our non-conforming approach. Let $\gamma_{c}$ denote the total number of degrees of freedom present in a problem with a conforming mesh (note the fact that in the elastic media there are two degrees of freedom per node). To fix the ideas, let suppose that the solid side requires a finer mesh discretization than the fluid side. Obviously, in the conforming case the domain with the finest mesh induces in the other domain a finer mesh than necessary. For this reason, for the same problem it is clear that the total number of degrees of freedom with our non-conforming technique, $\gamma_{n}$, is such that $\gamma_{n}<\gamma_{c}$. With this notation, we define the efficiency factor $\alpha$ as the gain of our non-conforming technique,

$$
\alpha:=1-\frac{\gamma_{n}}{\gamma_{c}},
$$




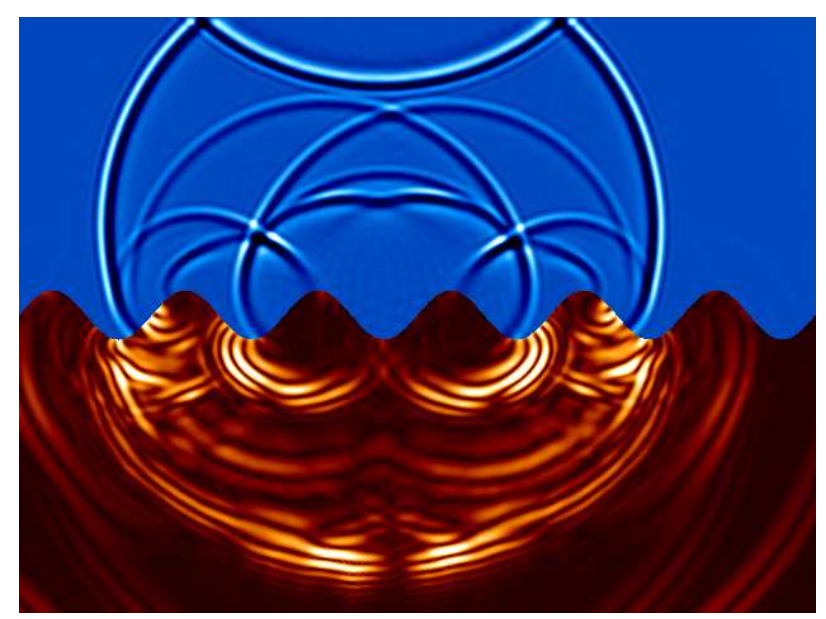

Figure 13: Snapshot of the reference test at time $t=1.57$ secs.

ranging from 0 to 1 , i.e., from minimum to maximum efficiency. This efficiency depends on several values, such as the physical dimension of the problem, $d$, the ratio between the size of the domain with the coarser mesh with respect to the size of the whole domain, $\left|\Omega_{\text {finer }}\right| /|\Omega|$, and the ratio between the typical linear size of the mesh discretization in both domains, $\beta_{h}=h_{f} / h_{s}$. With those definitions, we are able to be more precise about the formula in (22) for the efficiency in (22), claiming that

$$
\alpha:=1-\frac{\gamma_{n}}{\gamma_{c}} \simeq 1-\gamma_{c}^{-1}\left(\frac{\left|\Omega_{\text {finer }}\right|}{|\Omega|} \gamma_{c}+\frac{\left|\Omega_{\text {coarser }}\right|}{|\Omega|} \frac{1}{\beta_{h}^{d}} \gamma_{c}\right),
$$

Let us consider a concrete problem of reference to validate this estimation. As before, we consider a two-dimensional rectangular domain $\Omega=\Omega_{f} \cup \Omega_{s}$, with $\Omega=[0,64] \times[0,48]$, $\Omega_{s}=[0,64] \times[0,24]$ and $\Omega_{f}=[0,64] \times[24,48]$, so that $\left|\Omega_{s}\right|=\left|\Omega_{f}\right|=1 / 2|\Omega|$. Let suppose that we have a ratio of discretization $2: 1$ between the two subdomains, i.e., $\beta_{h}=h_{f} / h_{s}=2 h_{s} / h_{s}=2$. Thus, using all these values and formula (23), we expect to obtain the following efficiency for this problem

$$
\alpha_{p} \simeq 1-\gamma_{c}^{-1}\left(\frac{1}{2} \gamma_{c}+\frac{1}{2} \frac{1}{4} \gamma_{c}\right)=0.375
$$

Therefore, we estimate to save $37.5 \%$ of degrees of freedom for this problem with our non-conforming method. In Table 7 we report the numerical results obtained, for several mesh discretizations characterized by $h$. For each $h$-value considered, we compare $\gamma_{n}$ versus $\gamma_{c}$, and provide the efficiency obtained for each case as a function of $h, \alpha_{p}(h)$. Note the good agreement between the numerical values of $\alpha_{p}(h)$ and theoretical estimate $\alpha_{p}=0.375$. 


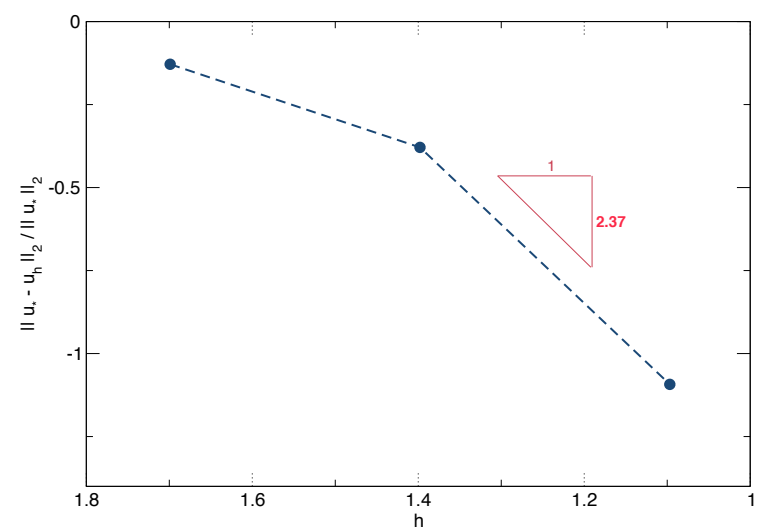

Figure 14: Convergence results for $P 1$-elements in log-log scale.

\begin{tabular}{|c|c|c|c|}
\hline \multirow{2}{*}{$h$} & \multicolumn{2}{|c|}{ Degrees of freedom } & \multirow{2}{*}{$\alpha_{p}(h)$} \\
\hline & $\gamma_{n}\left(h_{s}=h_{f}=h\right)$ & $\gamma_{c}\left(2 h_{s}=h_{f}=h\right)$ & \\
\hline 6.25 & 2,663 & 4,290 & 0.38461 \\
\hline 12.5 & 10,832 & 16,802 & 0.38425 \\
\hline 25 & 40,994 & 66,498 & 0.38353 \\
\hline 50 & 162,914 & 264,578 & 0.38209 \\
\hline \multirow[t]{2}{*}{100} & 649,538 & $1,055,490$ & 0.37925 \\
\hline & & & tical: $\alpha_{p}=0.375$ \\
\hline
\end{tabular}

Now we inspect the memory consumption related to the computational mesh as well as the computational time. In case of a ratio $4: 1$, the estimated efficiency is $\alpha_{p}=0 . \overline{4}$, which means that the amount of memory associated to the mesh must be reduced nearly by $44, \overline{4} \%$ with respect to the conforming mesh. In Table 7 we validate this estimation 255 for the same problem as before, where it is shown that our non-conforming method near halves the amount of memory consumption for several $h$-values considered, as expected. Note also the significant reduction of the computational time reported in the same table, for a simulation with final time $T_{\text {end }}=2.5$ seconds and time step $\Delta t=10^{-3}$ seconds. Note that we are relatively saving more computational time than memory. This behavior 260 is explained by the fact that the computational cost of MUMPS for both the factorization of the matrix and solving for the RHS, grows faster than linearly with respect to the number of unknowns. In this point it is worth mentioning that, despite the savings respect to the number of elements by our technique may be similar to those achieved by other non-conforming techniques, the latter introduce either additional degrees of freedom (as in Mortar-methods, for example) and/or additional parameters to control 


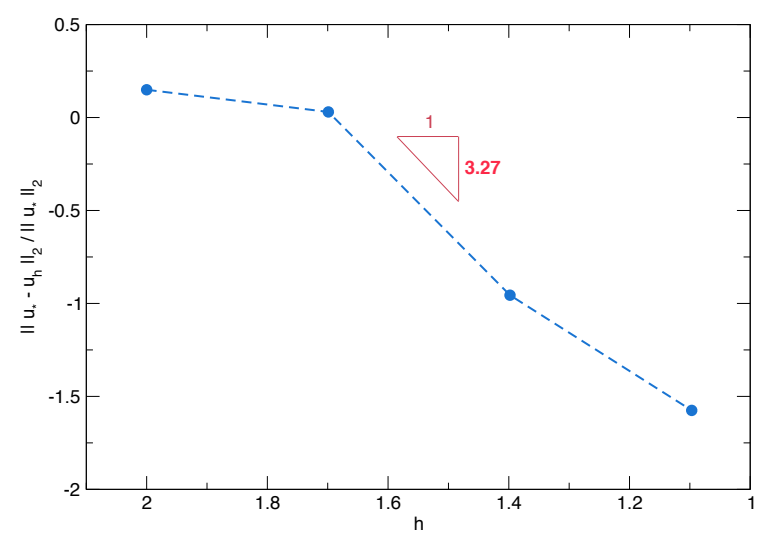

Figure 15: Convergence results for $P 2$-elements in log-log scale in log-log scale.

the accuracy (as in $[27,28,29]$ ).

\begin{tabular}{|c|c|c|c|c|c|c|}
\hline \multirow{2}{*}{$h$} & \multicolumn{3}{|c|}{ Memory size (MB) } & \multicolumn{3}{|c|}{ Time (s) } \\
\hline & Non-confor. & Confor. & Saved & Non-confor. & Confor. & Saved \\
\hline 6.25 & 63 & 122 & $48.4 \%$ & 3973 & 5036 & $58.4 \%$ \\
\hline 12.5 & 16 & 30 & $46.7 \%$ & 652 & 911 & $56.5 \%$ \\
\hline 25 & 3.7 & 7 & $47.1 \%$ & 154 & 207 & $57.3 \%$ \\
\hline \multicolumn{7}{|c|}{ Theoretical: $\alpha_{p}=0 . \overline{4}$} \\
\hline
\end{tabular}

Coupling more than two layers: the acoustic-acoustic and acoustic-elastic case

Finally, we show how the method performs under more complicated configurations, when more than two different media are considered. To describe our strategy, we consider here that the fluid domain $\Omega_{f}$ is divided in two sub-domains $\Omega_{a}$ and $\Omega_{w}$ (the air and the water for instance) such that $\Omega_{f}=\Omega_{a} \cup \Omega_{f}$. Note that the methodology can be extended without difficulty to the solid and to more than two domains. Denoting the pressure unknown by $q_{a}=q_{\mid \Omega_{a}}$ in the air layer and by $q_{w}=q_{\mid \Omega_{w}}$ in the water layer, the two continuity conditions to be imposed at their coupling interface $\Gamma_{a w}=\Omega_{a} \cap \Omega_{w}$ are

$$
q_{a}=q_{w},
$$

and

$$
\nabla \frac{1}{\rho_{a}} q_{a} \cdot \mathbf{n}=\frac{1}{\rho_{w}} \nabla q_{w} \cdot \mathbf{n} .
$$


Differently than in the acoustic-elastic coupling case, the first condition cannot be naturally imposed in the weak formulation. One possibility to circumvent this problem consists of introducing Lagrange multiplyers. However, we prefer to chose a Discontinuous Galerkin (DG)-like formulation instead, which is easier to implement in our method. Among the family of DG methods, we have based our solution methodology on the wellknown Interior Penalty DG method (IPDG) [34, 35, 36, 37]. We propose here to use continuous finite element in each subdomains and to couple the two subdomains using IPDG method. Thus, we search for solution $q \in L^{2}\left(\Omega_{f}\right)$ such that $q_{a}=q_{\mid \Omega_{a}} \in \mathcal{H}^{1}\left(\Omega_{a}\right)$ and $q_{w}=q_{\mid \Omega_{w}} \in \mathcal{H}^{1}\left(\Omega_{w}\right)$. In this case, the weak formulation corresponding to the two acoustic unknowns on each media and at their coupling interface reads as follows: $\forall t \in(0, T]$, find $q_{a}=q_{a}(t) \in Q_{a}$ and $q_{w}=q_{w}(t) \in Q_{w}$ such that

$$
\begin{aligned}
& \int_{\Omega_{a}} \rho_{a}^{-1} c_{a}^{-2} \frac{\partial^{2} q_{a}}{\partial t^{2}} w_{a} d \Omega+\int_{\Omega_{a}} \rho_{a}^{-1} \nabla q_{a} \cdot \nabla w_{a} d \Omega \\
& +\int_{\Omega_{w}} \rho_{w}^{-1} c_{w}^{-2} \frac{\partial^{2} q_{w}}{\partial t^{2}} w_{w} d \Omega+\int_{\Omega_{w}} \rho_{w}^{-1} \nabla q_{w} \cdot \nabla w_{w} d \Omega \\
& -\int_{\Gamma_{a w}} \frac{1}{2}\left(\frac{1}{\rho_{a}} \nabla q_{a}+\frac{1}{\rho_{w}} \nabla q_{w}\right)\left(w_{a}-w_{w}\right) \cdot \mathbf{n}_{\mathbf{a w}} d \Gamma \\
& -\int_{\Gamma_{a w}} \frac{1}{2}\left(q_{a}-q_{w}\right)\left(\frac{1}{\rho_{a}} \nabla w_{a}+\frac{1}{\rho_{w}} \nabla w_{w}\right) \cdot \mathbf{n}_{\mathbf{a w}} d \Gamma \\
& +\gamma \int_{\Gamma_{a w}}\left(q_{a}-q_{w}\right)\left(w_{a}-w_{w}\right) d \Gamma \\
-\int_{\Gamma_{i}} \frac{\partial \mathbf{v}}{\partial t} \cdot \mathbf{n}_{\mathbf{s}} w_{f} d \Gamma=\int_{\Omega_{f}} f w_{f} d \Omega, &
\end{aligned}
$$

for all test functions $w_{a} \in W_{a}$ and $w_{w} \in W_{w}$, where $Q_{a}$ and $W_{a}$ are defined as before

in (7) and (9) for the fluid media (we omit here the external boundaries for the sake of simplicity). Here, $\mathbf{n}_{\mathbf{a w}}$ denotes the normal vector from $\Omega_{a}$ to $\Omega_{w}$, and $\gamma$ is a positive penalizing coefficient that must be large enough in order to ensure the stability of the method, but not too large in order to not penalize the CFL condition. A careful analysis about the combination of our method with DG is out of the scope of this paper and thus reserved for future work. The variational equation in the solid remains unchanged.

First, we consider the case of coupling three different media by adding an air layer on top of the water layer. For the air layer, physical parameters are $\rho_{a}=1.204 \mathrm{~kg} \mathrm{~m}^{-3}$ for the density and $c_{a}=344 \mathrm{~m} \mathrm{~s}^{-1}$ for the acoustic-wave speed. Locating again the source in the fluid domain at $\mathbf{x}_{\mathbf{0}}=[29.0833,31]$, two snapshots for this test are shown in figures 16 and 17 , corresponding to physical times $t=1.4$ and $t=2.6$, respectively. Notice how the front wave emanating from the fluid media is being transmitted and reflected across the two non-conforming couplings, reaching the three media and behaving globally as expected.

As a second example, let us consider a more sophisticated configuration on which the three layers join together, corresponding for example to the physical situation where the shore line region is included. In Figure 18 we show part of the non-conforming mesh used for this test, emphasizing the region on which all the coupling boundaries meet. The ratio of points for the coupling between the fluid and the solid is approximately 2:1 (notice that it is not estrict), between the air and the fluid 2:1 as well, and 4:1 between the air 


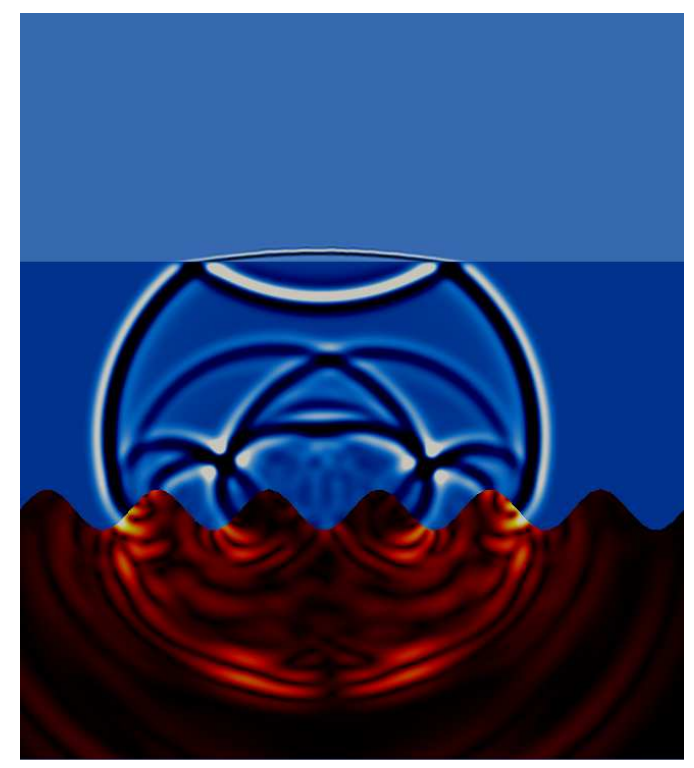

Figure 16: Snapshot of the simulation involving three different media, at time $t=1.4$. secs. The upper layer corresponds to the air acoustic layer, the middle to the fluid acoustic layer and the bottom to the solid elastic layer.

290 and the solid. Once again, locating the source in the fluid domain at $\mathbf{x}_{\mathbf{0}}=[29.0833,31]$, three snapshots for this test are shown in figures 19, 20 and 21, corresponding to the physical times $t=1.4, t=2.1$ and $t=3.6$, respectively. At time $t=1.4$, we illustrate the moment after the front of the wave reaches the first sharp point, between the fluid and the elastic media. At time $t=2.1$, the front wave has already crossed the point on which the three coupling boundaries intersect. And finally, at time $t=3.6$ we show the expected stable evolution of the simulation, on which the front wave has already reached all junctions and points of interest with the expected behavoir.

\section{Conclusions}

A new non-conforming numerical technique has been introduced, in the context of high-order Finite Element Methods for coupled wave propagation problems.

The advantage of this method with respect to other existing non-conforming techniques is based on the simplicity and generality of its application, since it is based on a geometrical adjustment between subdomains at its coupling interface, which is done at a preprocessing stage when the mesh is built. Another remarkable feature of this method is found on the fact that curved interfaces are geometrically approximated, in consonance, with the same accuracy as that of the finite element space discretization. That is to say, the method respects and takes the maximum possible advantage of the spatial discretization in a natural way. This has been possible by modeling the geometry of the interface with a unique spline, with the goal to project the edges of the elements 


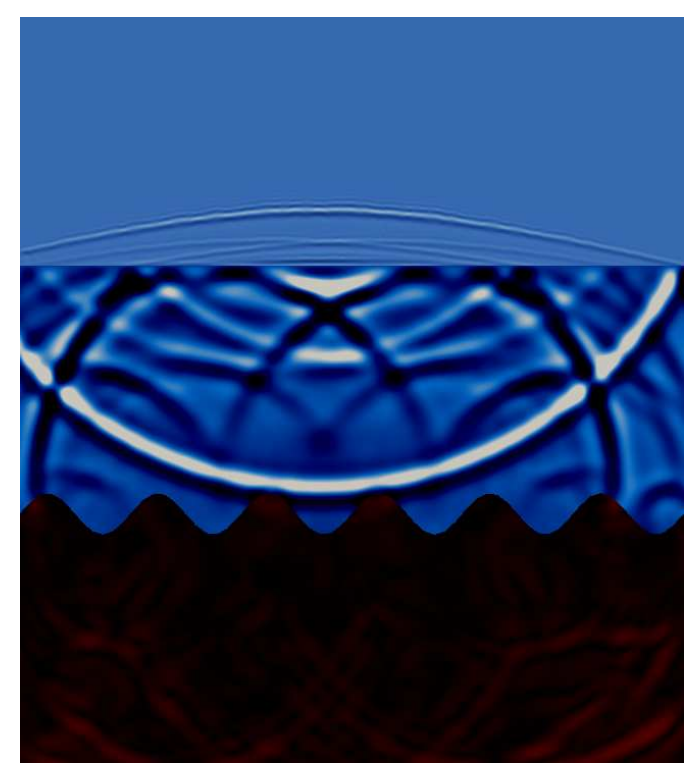

Figure 17: Snapshot of the simulation involving three different media, at time $t=2.6$. secs. The upper layer corresponds to the air acoustic layer, the middle to the fluid acoustic layer and the bottom to the solid elastic layer.

310 adjacent to the interface in such a way that it provides a perfect match between the coupled domains.

We have proved the conservation of a discrete energy for a second-order, Leap-frog discretization in time, both for explicit and implicit schemes. Moreover, we have proved that our coupling strategy does not penalize the CFL condition of the explicit scheme.

315 Of course, the study of this method with higher-order time schemes, in consonance with the space discretization, provides a direction for future work. To some extent, coupling very different meshes, although possible, may require the use of a local time stepping method. As far as the extension of our method to three dimensions, there is no theoretical difficulty. The main issues are simply of practical nature, related to the definition of two dimensional splines and the computations of the surface integrals.

Our proposed method is arbitrarily high-order in space and its convergence has been carefully studied. We have provided relevant numerical tests and compared the results with the well-established Spectral Element Method, showing an excellent agreement. Also, we have shown that our non-conforming technique reduces the size of the problem inversely proportional to the characteristic ratio of the non-conforming coupling, raised to the power of the dimension of the problem. This significant result amounts to a big saving in terms of memory consumption and computational time (decreasing faster than linearly with respect to the number of unknowns saved), a critical aspect in the context of large scale wave propagation problems.

330 Another remarkable feature of this method is found on its suitability to be extended to any methodology based on the Finite Element Method. In the particular case of the Spectral Element Method, widely used in geophysical applications, no extra work has to 


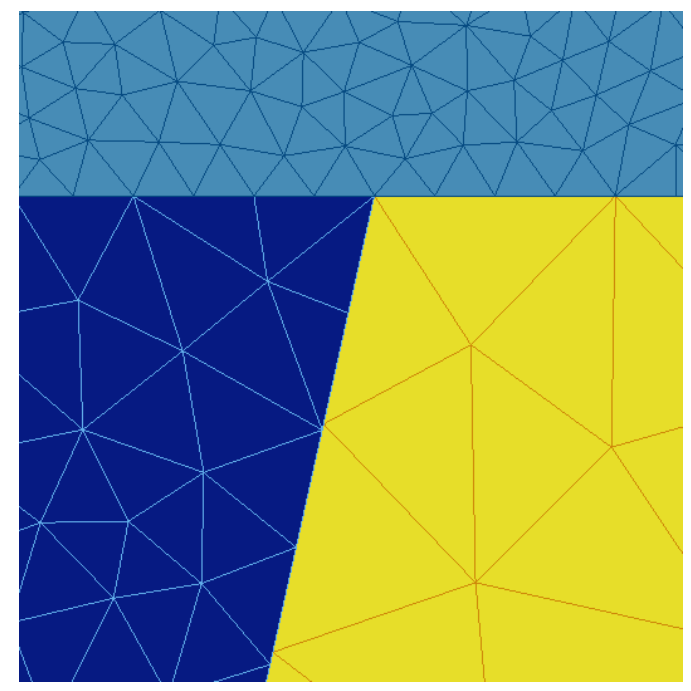

Figure 18: Illustration of the non-conforming mesh used in the case of a three layers simulation including the shore line region, zooming in on the point on which the coupling boundaries intersect.

be introduced once the coupling stage is completed. The same argument applies for the family of Discontinuous Galerkin methods and Isogeometric Analysis. Interestingly, as it occurs in Mortar-methods, the use of Discontinuous Galerkin methods in our proposed technique (as we have shown for the case of coupling two acoustics media) allows to obtain algebraic block systems. This feature will certainly be worth investigating further for building efficient solvers for very large systems and parallel computations.

Finally, we remark the general applicability of this non-conforming technique to any multiphysics, multiscale phenomena formulated as a coupled problem.

\section{Acknowledgements}

The first author acknowledges support from Spanish Ministry under Grant No. FPDI2013-17098 and from the Conseil Régional d'Aquitaine under Grant 2011-6931. This work has been partly supported by FP7 IRSES Marie-Curie HPC-GA. We thank Lakhdar Remaki (BCAM-Basque Center of Applied Mathematics), for interesting discussions and providing help in the analysis of numerical results.

[1] Z. Chen, G. Hofstetter, H. Mang, A Galerkin-type BE-FE formulation for elasto-acoustic coupling, Computer methods in applied mechanics and engineering 152 (1) (1998) 147-155.

[2] L. Gaul, D. Brunner, M. Junge, Simulation of elastic scattering with a coupled FMBE-FE approach, in: Recent Advances in Boundary Element Methods, Springer, 2009, pp. 131-145.

[3] M. Fischer, L. Gaul, Fast BEM-FEM mortar coupling for acoustic-structure interaction, International Journal for Numerical Methods in Engineering 62 (12) (2005) 1677-1690.

[4] A. Márquez, S. Meddahi, V. Selgas, A new BEM-FEM coupling strategy for two-dimensional fluidsolid interaction problems, Journal of Computational Physics 199 (1) (2004) 205-220.

355 [5] S. Schneider, FE/FMBE coupling to model fluid-structure interaction, Int. J. Numer. Meth. Engng. 76 (13) (2008) 2137-2156. 


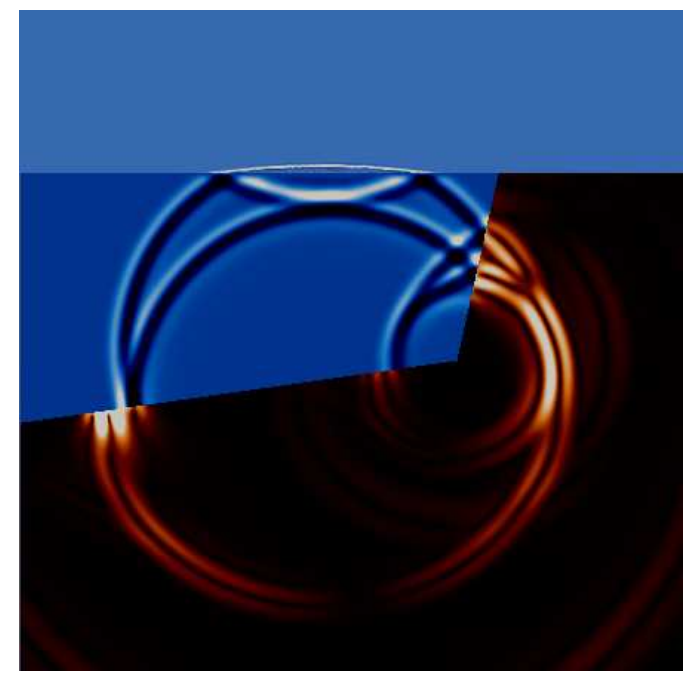

Figure 19: Snapshot of the simulation involving three different media at time $t=1.4$. secs. The front wave passes through the fluid-elastic coupling.

[6] A. El Kacimi, O. Laghrouche, Numerical modelling of elastic wave scattering in frequency domain by the partition of unity finite element method, International journal for numerical methods in engineering 77 (12) (2009) 1646-1669.

[7] T. Huttunen, J. Kaipio, P. Monk, An ultra-weak method for acoustic fluid-solid interaction, Journal of Computational and Applied Mathematics 213 (1) (2008) 166-185.

[8] P. Massimi, R. Tezaur, C. Farhat, A discontinuous enrichment method for three-dimensional multiscale harmonic wave propagation problems in multi-fluid and fluid-solid media, International Journal for Numerical Methods in Engineering 76 (3) (2008) 400-425.

[9] H. Barucq, R. Djellouli, E. Estecahandy, Efficient DG-like formulation equipped with curved boundary edges for solving elasto-acoustic scattering problems, International Journal for Numerical Methods in Engineering 98 (10) (2014) 747-780.

[10] J. Virieux, P-SV wave propagation in heterogeneous media: Velocity-stress finite-difference method, Geophysics 51 (4) (1986) 889-901.

370 [11] J. O. Robertsson, A numerical free-surface condition for elastic/viscoelastic finite-difference modeling in the presence of topography, Geophysics 61 (6) (1996) 1921-1934.

[12] G. Seriani, 3-D large-scale wave propagation modeling by spectral element method on Cray T3E multiprocessor, Computer Methods in Applied Mechanics and Engineering 164 (1) (1998) 235-247.

[13] G. Seriani, E. Priolo, Spectral element method for acoustic wave simulation in heterogeneous media, Finite elements in analysis and design 16 (3) (1994) 337-348.

[14] D. Komatitsch, J. Tromp, Introduction to the spectral element method for three-dimensional seismic wave propagation, Geophysical journal international 139 (3) (1999) 806-822.

[15] D. Komatitsch, J.-P. Vilotte, The spectral element method: an efficient tool to simulate the seismic response of $2 \mathrm{D}$ and $3 \mathrm{D}$ geological structures, Bulletin of the seismological society of America 88 (2) (1998) 368-392.

[16] E. Chaljub, D. Komatitsch, J.-P. Vilotte, Y. Capdeville, B. Valette, G. Festa, Spectral-element analysis in seismology, Advances in Geophysics 48 (2007) 365-419.

[17] D. Komatitsch, C. Barnes, J. Tromp, Wave propagation near a fluid-solid interface: A spectralelement approach, Geophysics 65 (2) (2000) 623-631.

[18] M. Käser, M. Dumbser, A highly accurate discontinuous Galerkin method for complex interfaces between solids and moving fluids, Geophysics 73 (3) (2008) T23-T35.

[19] C. Bernardi, A new nonconforming approach to domain decomposition: the mortar element method, Nonliner Partial Differential Equations and Their Applications. 


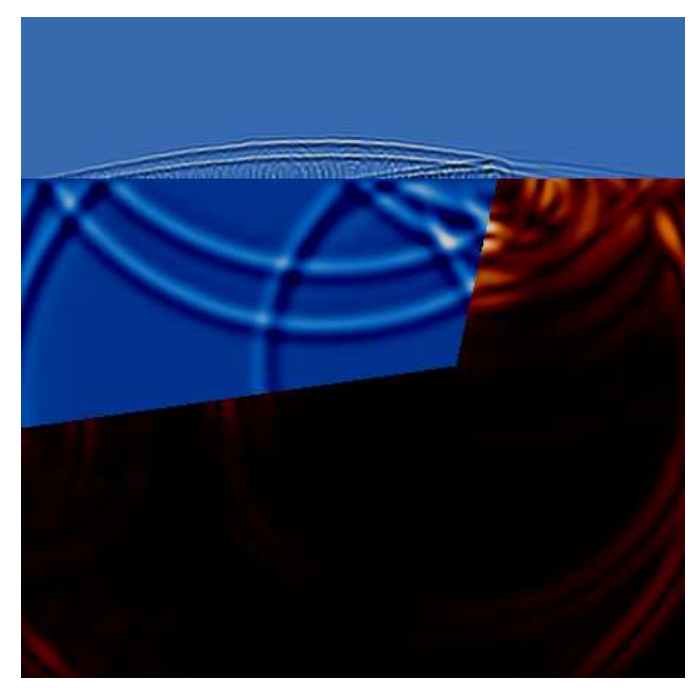

Figure 20: Snapshot of the simulation involving three different media at time $t=2.1$. secs. The front wave passes through the fluid-air coupling.

[20] F. B. Belgacem, The mortar finite element method with Lagrange multipliers, Numerische Mathematik 84 (2) (1999) 173-197.

[21] B. I. Wohlmuth, A mortar finite element method using dual spaces for the Lagrange multiplier, SIAM Journal on Numerical Analysis 38 (3) (2000) 989-1012.

[22] Y. Achdou, C. Japhet, Y. Maday, F. Nataf, A new cement to glue non-conforming grids with Robin interface conditions: The finite volume case, Numerische Mathematik 92 (4) (2002) 593-620.

[23] P. Antonietti, I. Mazzieri, A. Quarteroni, F. Rapetti, Non-conforming high order approximations of the elastodynamics equation, Computer Methods in Applied Mechanics and Engineering 209âĂŞ212 (0) (2012) $212-238$.

[24] E. Chaljub, Y. Capdeville, J.-P. Vilotte, Solving elastodynamics in a fluid-solid heterogeneous sphere: a parallel spectral element approximation on non-conforming grids, Journal of Computational Physics 187 (2) (2003) 457-491.

[25] R. Madec, D. Komatitsch, J. Diaz, Energy-conserving local time stepping based on high-order finite elements for seismic wave propagation across a fluid-solid interface, Computer Modeling in Engineering and Sciences (CMES) 14 (2) (2009) 163.

[26] J. Diaz, P. Joly, Robust high order non-conforming finite element formulation for time domain fluid-structure interaction, Journal of Computational Acoustics 13 (03) (2005) 403-431.

[27] R. K. Jaiman, X. Jiao, P. H. Geubelle, E. Loth, Assessment of conservative load transfer for fluid-solid interface with non-matching meshes, International Journal for Numerical Methods in Engineering 64 (15) (2005) 2014-2038.

[28] R. Jaiman, X. Jiao, P. Geubelle, E. Loth, Conservative load transfer along curved fluid-solid interface with non-matching meshes, Journal of Computational Physics 218 (1) (2006) 372 - 397.

[29] R. Jaiman, P. Geubelle, E. Loth, X. Jiao, Combined interface boundary condition method for unsteady fluid-structure interaction, Computer Methods in Applied Mechanics and Engineering $200(1-4)(2011)$ 27-39.

[30] L. C. Wilcox, G. Stadler, C. Burstedde, O. Ghattas, A high-order discontinuous Galerkin method for wave propagation through coupled elasticâĂŞacoustic media, Journal of Computational Physics 229 (24) (2010) $9373-9396$.

[31] J. E. Kozdon, L. C. Wilcox, Provably stable, general purpose projection operators for high-order finite difference methods, arXiv preprint arXiv:1410.5746.

[32] P. R. Amestoy, I. S. Duff, J.-Y. L'Excellent, J. Koster, A fully asynchronous multifrontal solver using distributed dynamic scheduling, SIAM Journal on Matrix Analysis and Applications 23 (1) 


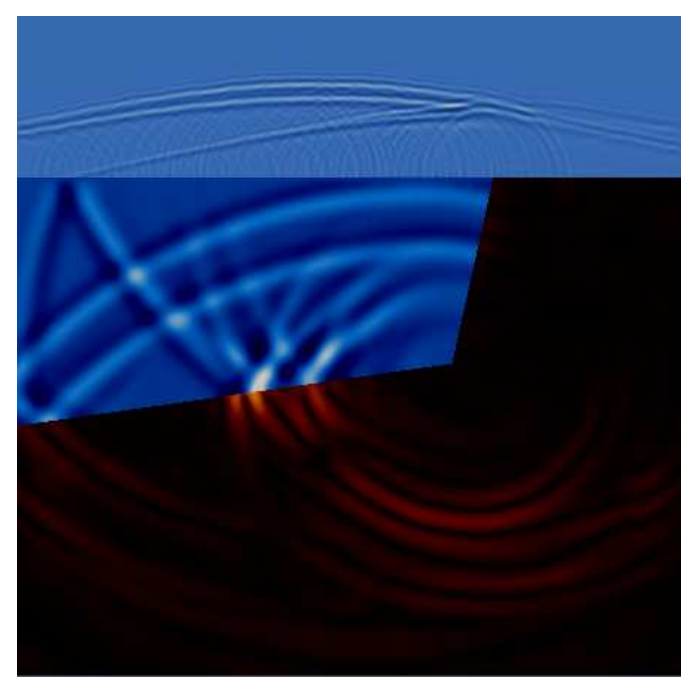

Figure 21: Snapshot of the simulation involving three different media at time $t=3.6$. secs. Stable evolution of the simulation on which waves are present in all three media.

(2001) 15-41.

[33] C. Geuzaine, J.-F. Remacle, Gmsh: A 3-D finite element mesh generator with built-in pre- and postprocessing facilities, International Journal for Numerical Methods in Engineering 79 (11) (2009) $1309-1331$.

[34] M. Ainsworth, P. Monk, W. Muniz, Dispersive and dissipative properties of discontinuous Galerkin finite element methods for the second-order wave equation, Journal of Scientific Computing 27 (1-3) (2006) 5-40.

[35] M. J. Grote, A. Schneebeli, D. Schötzau, Discontinuous Galerkin finite element method for the wave equation, SIAM J. Numer. Anal. 44 (6) (2006) 2408-2431 (electronic).

430 [36] M. J. Grote, A. Schneebeli, D. Schötzau, Interior penalty discontinuous Galerkin method for Maxwell's equations: Energy norm error estimates, Journal of Computational and Applied Mathematics 204 (2) (2007) 375 - 386, special Issue: The Seventh International Conference on Mathematical and Numerical Aspects of Waves (WAVES'05).

[37] M. J. Grote, A. Schneebeli, D. Schötzau, Interior penalty discontinuous Galerkin method for Maxwell's equations: Optimal $L^{2}$-norm error estimates, IMA J. Numer. Anal. 28 (3) (2008) 440-468, submitted. 